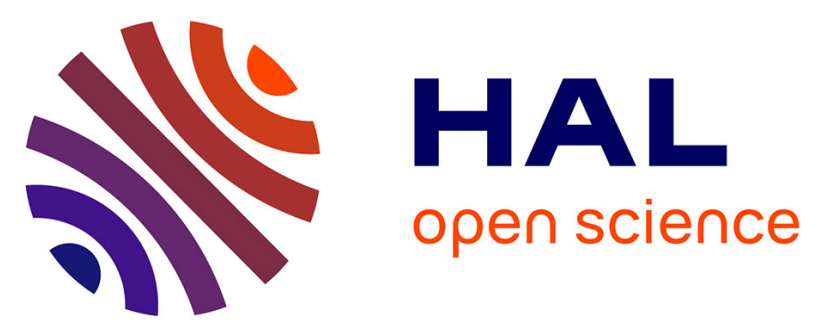

\title{
Electronically excited rubidium atom in helium clusters and films. II. Second excited state and absorption spectrum
}

\author{
Markku Leino, Alexandra Viel, Robert E. Zillich
}

\section{- To cite this version:}

Markku Leino, Alexandra Viel, Robert E. Zillich. Electronically excited rubidium atom in helium clusters and films. II. Second excited state and absorption spectrum. Journal of Chemical Physics, 2011, 134 (2), pp.024316. 10.1063/1.3528936 . hal-00711625

\section{HAL Id: hal-00711625 \\ https://hal.science/hal-00711625}

Submitted on 10 Jul 2017

HAL is a multi-disciplinary open access archive for the deposit and dissemination of scientific research documents, whether they are published or not. The documents may come from teaching and research institutions in France or abroad, or from public or private research centers.
L'archive ouverte pluridisciplinaire HAL, est destinée au dépôt et à la diffusion de documents scientifiques de niveau recherche, publiés ou non, émanant des établissements d'enseignement et de recherche français ou étrangers, des laboratoires publics ou privés. 


\title{
Electronically excited rubidium atom in helium clusters and films. II. Second excited state and absorption spectrum
}

\author{
Markku Leino, ${ }^{1}$ Alexandra Viel, ${ }^{1}$, a) and Robert E. Zillich ${ }^{2}$ \\ ${ }^{1}$ Institut de Physique de Rennes, UMR 6251, CNRS \& Université de Rennes 1, F-35042 Rennes, France \\ ${ }^{2}$ Institute for Theoretical Physics, Johannes Kepler Universität Linz, Altenbergerstraße 69, A-4040 Linz, Austria
}

(Received 14 October 2010; accepted 1 December 2010; published online 12 January 2011)

Following our work on the study of helium droplets and film doped with one electronically excited rubidium atom $\mathrm{Rb}^{*}\left({ }^{2} \mathrm{P}\right)$ [M. Leino, A. Viel, and R. E. Zillich, J. Chem. Phys. 129, 184308 (2008)], we focus in this paper on the second excited state. We present theoretical studies of such droplets and films using quantum Monte Carlo approaches. Diffusion and path integral Monte Carlo algorithms combined with a diatomics-in-molecule scheme to model the nonpair additive potential energy surface are used to investigate the energetics and the structure of $\mathrm{Rb}^{*} \mathrm{He}_{n}$ clusters. Helium films as a model for the limit of large clusters are also considered. As in our work on the first electronic excited state, our present calculations find stable $\mathrm{Rb}^{*} \mathrm{He}_{n}$ clusters. The structures obtained are however different with a $\mathrm{He}-\mathrm{Rb}^{*}-\mathrm{He}$ exciplex core to which more helium atoms are weakly attached, preferentially on one end of the core exciplex. The electronic absorption spectrum is also presented for increasing cluster sizes as well as for the film. (c) 2011 American Institute of Physics. [doi:10.1063/1.3528936]

\section{INTRODUCTION}

Helium-4 nanodroplets ${ }^{1-3}$ are unique spectroscopic tools to probe atoms and molecules at low temperatures. Electronic spectroscopy as well as the goal to study chemical reactions activated by light in this superfluid quantum matrix, motivate much of the experimental and theoretical work. Because of the relative simplicity of their electronic structure, with a single electron in the valence shell, alkali atoms are favored study objects.

Due to the weak van der Waals interaction with helium, dopant alkali atoms reside on the surface of helium droplets where they are bound ${ }^{4}$ with binding energies around $10 \mathrm{~K}$ (Ref. 5). When the adsorbed alkali atom is electronically excited, an alkali-helium exciplex can form. ${ }^{6-13}$ The potential of the first three electronically excited states of the $\mathrm{Rb}^{*}-\mathrm{He}$ dimer differ in shape: $1^{2} \Pi_{1 / 2}$ presents two attractive wells separated by a barrier, $1^{2} \Pi_{3 / 2}$ exhibits one attractive well, and $2^{2} \Sigma_{1 / 2}$ is only very slightly attractive at large interatomic distances and does not support bound states. Thus, the first two states in principle permit formation of exciplexes ${ }^{9}$ $\mathrm{Rb}^{*} \mathrm{He}_{m}$ while the third state is purely destructive. Exciplex formation releases enough energy to cause desorption ${ }^{6,9,14,15}$ of the $\mathrm{Rb}^{*} \mathrm{He}_{m}$ exciplex. However, it is possible to excite $\mathrm{Rb}$ atoms adsorbed on the surface of a ${ }^{4} \mathrm{He}$ droplet without exciplex formation and subsequent desorption, as described experimentally in Ref. 15.

In Ref. 16, hereafter noted paper I, we studied the vibrational ground state of one electronically excited $\mathrm{Rb}$ atom $\left(1^{2} \Pi_{1 / 2}\right.$ state) and a ${ }^{4} \mathrm{He}$ cluster. We showed that a ring of seven helium atoms forms around $\mathrm{Rb}^{*}$, thus leading to the conclusion that $\mathrm{Rb}^{*} \mathrm{He}_{7}$ is the largest stable exciplex. For the second electronic excited state, $1^{2} \Pi_{3 / 2}$, theoretical work ${ }^{17-19}$ showed that a maximum number of two helium atoms are strongly bound to the alkali atom. In this paper, we confirm

\footnotetext{
a)Electronic mail: alexandra.viel@univ-rennes1.fr.
}

this conclusion in the case of $\mathrm{Rb}^{*}$ alkali by calculating the exact ground state energies up to $n=8$ helium atoms by importance sampling diffusion Monte Carlo (DMC) and pathintegral Monte Carlo (PIMC) simulations.

Theoretical modeling of the dynamics after the excitation of an alkali atom adsorbed on a ${ }^{4} \mathrm{He}$ droplet is a very challenging problem that requires to resort to approximations. Reho et al. ${ }^{8}$ proposed a one-dimensional model to estimate the exciplex formation time for various alkali atoms while Pacheco et al. ${ }^{20}$ used a quantum-classical modeling for $\mathrm{Li}$ attached to $\mathrm{He}_{99}$. The dynamics of $\mathrm{K}^{*}$ desorption from $\mathrm{He}$ clusters has been studied by Takayanagi and Shiga ${ }^{21}$ using an approximation based on semiclassical path integral centroid molecular dynamics. In paper I, we proposed a "vertical Monte Carlo (MC) transition" in the course of a quantum Monte Carlo simulation in order to gain some insight into the dynamics after excitation to the $1^{2} \Pi_{1 / 2}$ state. Although this approach is a purely computational technique and does not correspond to real dynamics, it allowed us to get a better understanding of this multidimensional problem. In particular, we found the metastable surface state of $\mathrm{Rb}^{*}$ on helium droplets, in agreement with experiments. ${ }^{15,22}$ In the present work, we use the same vertical MC technique to study the excitation of $\mathrm{Rb}$ on $\mathrm{He}$ clusters and films to the $1^{2} \Pi_{3 / 2}$ state. We find formation of the $\mathrm{Rb}{ }^{*} \mathrm{He}$ exciplexes, but not of $\mathrm{Rb}^{*} \mathrm{He}_{2}$.

Measurement and analysis of the electronic absorption and emission spectrum of $\mathrm{RbHe}_{m}$ provide valuable information on the complicated underlying alkali-helium interaction potential as well as on the dynamics of exciplex formation. Excitation spectra of alkali-helium exciplexes have been recorded in solid ${ }^{4} \mathrm{He},{ }^{19,23,24}$, liquid ${ }^{4} \mathrm{He},{ }^{25-27}{ }^{4} \mathrm{He}$ gas, ${ }^{11,14}$ and on ${ }^{4} \mathrm{He}$ nanodroplets. ${ }^{9,28-31}$ We calculate an approximated absorption spectrum of $\mathrm{Rb}$ for He cluster sizes ranging from $n=2$ to $n=300$ and for $\mathrm{Rb}$ on a flat He film. The spectra consist of several broad spectral lines with a linewidth that is strongly dependent on the cluster size. 
This paper is structured as follows: in Sec. II we briefly describe the methodology (DMC and PIMC). In Sec. III we present energetics and structure of small droplets, Sec. IV is dedicated to the study of $\mathrm{Rb}$ and $\mathrm{Rb}^{*}$ on a ${ }^{4} \mathrm{He}$ surface, and in Sec. V we present our PIMC results for electronic absorption spectra.

\section{THEORETICAL APPROACH}

\section{A. Description of the system}

The description of the system has been detailed in paper I. Shortly, the interaction potential between the $n$ helium atoms $(\mathrm{He})$ and the excited rubidium atom $\left(\mathrm{Rb}^{*}\right)$ is given by the eigenvalues of a $6 \times 6$ complex matrix, which relies on the diatomics-in-molecules (DIM) approximation ${ }^{30,32}$ to account for the anisotropy of $\mathrm{Rb}^{*}$ interacting with multiple helium atoms and which contains the spin-orbit interaction. ${ }^{33}$ Note that a typo is present in Eq. (2) of paper I: $\Delta_{\text {SO }}$ has to be replaced by $\Delta_{\text {SO }} / 3$ in the matrix, where $\Delta_{\text {SO }}=237.6 \mathrm{~cm}^{-1}$ is the spin-orbit splitting of $\mathrm{Rb}^{*}$. The Hamiltonian, $\hat{H}$, contains the kinetic energy terms for all particles, the $\mathrm{He}-\mathrm{He}$ interaction (Aziz HFD-B potential ${ }^{34}$ ) and the interaction between the helium atoms and the excited alkali atom in the DIM approximation.

In paper I, we focused on the lowest potential energy surface corresponding to the $1^{2} \Pi_{1 / 2}$ state, using spectroscopic notation which is, strictly speaking, valid only for the $\mathrm{Rb}^{*} \mathrm{He}$ dimer. In the present work, we study $\mathrm{Rb}^{*} \mathrm{He}_{n}$ interacting via the second potential energy surface (the $\mathrm{Rb}^{*} \mathrm{He}$ interaction for the third electronic state is essentially repulsive and no cluster can be formed). This second electronic state corresponds to a $1^{2} \Pi_{3 / 2}$ state in the dimer case. The full vibrational Schrödinger equation is expressed using the $3 n+3$ Cartesian coordinates of the $n \mathrm{He}$ atoms and of the $\mathrm{Rb}$ atom. The underlying $\mathrm{Rb}^{*}-\mathrm{He}$ interaction potential curves needed in the DIM model are taken from Pascale's work ${ }^{35,36}$ to stay consistent with the work performed in paper I. Though there exists some criticism of the accuracy of Pascale's potentials ${ }^{11,13}$ they seem physically relevant and provide good correspondence between theoretical emission (absorption) and observed spectra. ${ }^{9}$

\section{B. Diffusion Monte Carlo}

We use the importance sampling (or guided) diffusion Monte Carlo (ISDMC) algorithm to obtain the ground state of $\mathrm{Rb}^{*} \mathrm{He}_{n}$ clusters. In paper I, we used the standard first order implementation of the ISDMC algorithm, while here we employ the second order scheme which allows to use larger time steps at the cost of more evaluations of the drift force per time step.

In ISDMC, the $\mathcal{N}=3 n+3$ dimensional ground state wavefunction $\Phi_{0}(\mathcal{X})$ is not sampled directly, but a guiding wave function $\Psi_{T}(\mathcal{X})$ is introduced to improve sampling efficiency. The product $f(\mathcal{X}, \tau)=\Phi(\mathcal{X}, \tau) \Psi_{T}(\mathcal{X})$ is evolved in imaginary time $\tau$ toward the equilibrium $\Phi_{0}(\mathcal{X}) \Psi_{T}(\mathcal{X})$ according to the diffusion equation

$$
\begin{aligned}
\frac{\partial f}{\partial \tau} & =\left[\sum_{j=1}^{\mathcal{N}}\left(D_{j} \frac{\partial^{2}}{\partial \mathcal{X}_{j}^{2}}-D_{j} \frac{\partial F_{j}}{\partial \mathcal{X}_{j}}\right)-\left(E_{l}-E_{\mathrm{ref}}\right)\right] f, \\
& \equiv[T+D+E] f .
\end{aligned}
$$

In the above equation, $D_{j}=\hbar^{2} / 2 M_{\alpha}$ where $\alpha=\mathrm{Rb}$ or $\alpha$ $=$ He depending on whether $\mathcal{X}_{j}$ is the Cartesian coordinate of the $\mathrm{Rb}^{*}$ atom or of a ${ }^{4} \mathrm{He}$ atom, and $\mathcal{X} \in \mathbb{R}^{3 n+3}$. The local energy is defined as $E_{l}(\mathcal{X})=\Psi_{T}(\mathcal{X})^{-1} \hat{H} \Psi_{T}(\mathcal{X})$ and the quantum force is given by $F_{j}(\mathcal{X})=\left(\partial / \partial \mathcal{X}_{j}\right) \ln \left|\Psi_{T}(\mathcal{X})\right|^{2}$. The energies are given with respect to the reference energy $E_{\text {ref }}$ chosen such that the vibrational ground state has zero energy. The formal solution to Eq. (1) can be expressed in terms of the imaginary time propagator

$$
\begin{aligned}
f(\tau) & =\exp [-\tau(T+D+E)] f(0) \\
& =(\exp [-\Delta \tau(T+D+E)])^{m} f(0),
\end{aligned}
$$

where we have split a long time step $\tau$ into small steps $\Delta \tau=\tau / m$. Since the propagator cannot be evaluated exactly in general, it has to be approximated. For small time steps, a second order Trotter expansion can be used in Eq. (2)

$$
\begin{aligned}
& e^{-\Delta \tau(T+D+E)} \\
& \quad=e^{-\frac{\Delta \tau}{2} E} e^{-\frac{\Delta \tau}{2} T} e^{-\Delta \tau D} e^{-\frac{\Delta \tau}{2} T} e^{-\frac{\Delta \tau}{2} E}+O\left(\Delta \tau^{3}\right) .
\end{aligned}
$$

All the separate propagators of Eq. (3) can be obtained analytically in coordinate space. Note that this factorization of Eq. (3) is not unique. This choice is based on the work of Chin ${ }^{37}$ in which various second order DMC schemes have been studied, and on the implementation presented in Ref. 38. Equation (3) leads to the second order update algorithm of the coordinates of a walker from $\mathcal{X}_{i}$ to $\mathcal{X}_{i}^{\prime}$

$$
\begin{aligned}
& \mathcal{Y}_{i}=\mathcal{X}_{i}+2^{-1 / 2} \xi_{i} \\
& \mathcal{X}_{i}^{\prime}=\mathcal{Y}_{i}+2 \Delta \tau F_{i}^{\prime}\left(\mathcal{Y}+\Delta \tau F^{\prime}(\mathcal{Y})\right)+2^{-1 / 2} \xi_{i}^{\prime},
\end{aligned}
$$

where $\xi_{i}$ and $\xi_{i}^{\prime}$ are random numbers sampled from a Gaussian distribution of width $\sqrt{2 D_{i} \Delta \tau}$ and $F_{i}^{\prime} \equiv D_{i} F_{i}$. The propagator $\exp [-\Delta \tau E]$ in Eq. (3) enters as a weight of the walkers. In this work, we use an implementation which relies on a combination of weights and branching, resulting in a fixed ensemble size similar to the implementation used in Ref. 39 and developed in Ref. 40.

In the limit of an infinite number of walkers and zero time step, the iteration of Eq. (2) yields the stationary distribution $\Phi_{0}(\mathcal{X}, \tau) \Psi_{T}(\mathcal{X})$ and the ground state energy $E_{0}$. Within the second order DMC algorithm used in this work, both ways to estimate $E_{0}$, namely the growth estimator and the average of the local energy, converge quadratically with the imaginary time step $\Delta \tau$. Thus, a quadratic extrapolation to $\Delta \tau \rightarrow 0$ yields the correct $\Delta \tau=0$ limit of $E_{0}$.

In addition to the time step bias, a systematic bias is introduced by the use of a finite number of walkers $N_{\text {wlk }}<\infty$. This ensemble size bias can be estimated as explained in Refs. 41-43, and 44 by

$$
E_{0}^{N_{\mathrm{wlk}}}=E_{0}+c_{\mathrm{wlk}} N_{\mathrm{wlk}}^{-1}+\cdots .
$$


Thus, an extrapolation to infinite number of walkers is carried out by several simulations, each with different ensemble size $N_{\text {wlk }}$. Note that there are other approaches to avoid the extra computations required by this extrapolation scheme. ${ }^{41,45}$

As in paper I, we employed a modification of the guiding wave function that was found to be successful in studying doped helium clusters ${ }^{46}$ and that does not depend on the $\mathrm{Rb}$ atom coordinates

$$
\Psi_{T}=\prod_{i \in \mathrm{He}} \chi^{\mathrm{He}}\left(r_{i}\right) \prod_{i, j \in \mathrm{He}} \Phi^{\mathrm{He}-\mathrm{He}}\left(r_{i j}\right),
$$

where $r_{i}$ is the distance from $i$ th He atom to the center of mass of the helium atoms and $r_{i j}$ is the distance between the $i$ th and the $j$ th $\mathrm{He}$ atom. Note that in paper I, $r_{i}$ was incorrectly defined as the distance between $\mathrm{He}$ and $\mathrm{Rb}$ atom. The functions in Eq. (6) are

$$
\begin{aligned}
& \chi^{\mathrm{He}}(r)=\left\{1+\exp \left[f_{1}\left(r-f_{2}\right)\right]\right\}^{-1}, \\
& \Phi^{\mathrm{He}-\mathrm{He}}(r)=\exp \left(-a r^{-5}\right) .
\end{aligned}
$$

The Fermi type function, $\chi$, ensures asymptotic exponential decay (thus preventing unphysical evaporation of He atoms from the cluster) without any structural bias at short range, and the Jastrow part, $\Phi$, ensures that two atoms will not overlap.

In this work, we perform simulations for several $\Delta \tau$ from 400 a.u. down to 10 a.u. in order to extrapolate to the $\Delta \tau=0$ limit. The ensemble size bias is corrected by extrapolation, using simulations with walker populations ranging from $N_{\text {wlk }}=1000$ to $N_{\text {wlk }}=8000$, which has been found to be adequate for $\mathrm{Rb}^{*} \mathrm{He}_{n}$ clusters. The value of the parameter $a$ has been fixed at 500 like in our previous work. We found that the parameters of the Fermi function have an influence on the value of the energies obtained at a given time step and ensemble size, but that the extrapolated (and relevant) values are independent of the parameters as long as they are physically reasonable. We found that $f_{1}=1$ and $f_{2}=30$ in a.u. enable efficient computations of the zero point energies for the cluster sizes studied here.

\section{Path integral Monte Carlo}

An overview of the PIMC method for finite temperature simulations was given in paper I. We repeat here only the most important details regarding the time step bias; for a full review of the PIMC method for bosons see Ref. 47.

The PIMC method samples the configuration space representation of the many-body density matrix $\rho\left(\mathcal{X}, \mathcal{X}^{\prime} ; \beta\right)$ $=\left\langle\mathcal{X}\left|e^{-\beta \hat{H}}\right| \mathcal{X}^{\prime}\right\rangle$, where $\beta=1 / k_{\mathrm{B}} T$ corresponds to an imaginary time interval. For the evaluation of $\rho\left(\mathcal{X}, \mathcal{X}^{\prime} ; \beta\right), \beta$ needs to be split into smaller time steps $\Delta \tau=\beta / M$ leading to new coordinates at intermediate "time slices,"

$$
\begin{aligned}
\rho\left(\mathcal{X}_{0}, \mathcal{X}_{M} ; \beta\right)= & \int d \mathcal{X}_{1} \cdots \mathcal{X}_{M-1} \rho\left(\mathcal{X}_{0}, \mathcal{X}_{1} ; \Delta \tau\right) \cdots \\
& \cdots \rho\left(\mathcal{X}_{M-1}, \mathcal{X}_{M} ; \Delta \tau\right) .
\end{aligned}
$$

Here $\left(\mathcal{X}_{0}, \ldots, \mathcal{X}_{M}\right)$ is a discretized path in imaginary time. For sufficiently small $\Delta \tau, \rho\left(\mathcal{X}_{0}, \mathcal{X}_{1} ; \Delta \tau\right)$ can be approximated in various ways. As in Ref. 48, we use the pair density approximation $^{47}$ for the He-He interaction, which has been found to allow time steps up to $\Delta \tau=(40 \mathrm{~K})^{-1}$. However, the pair density approximation would be too complicated to implement for interactions like $\mathrm{Rb}^{*}-\mathrm{He}_{n}$ that is not pair-wise additive. Therefore we resort to the Trotter approximation $e^{-\Delta \tau \hat{H}}=e^{-\Delta \tau \hat{V} / 2} e^{-\Delta \tau \hat{T}} e^{-\Delta \tau \hat{V} / 2}+\mathrm{O}\left(\Delta \tau^{3}\right)$, for the $\mathrm{Rb}^{*}-\mathrm{He}_{n}$ interaction part of the potential term. This leads to a large quadratic time step bias of the energy and other properties due to the steepness of the $\mathrm{Rb}^{*}-\mathrm{He}_{n}$ interaction around the potential minimum (see Sec. III A). Much care has to be exercised to make sure that the $\Delta \tau$ bias is small. In the appendix, we discuss the $\Delta \tau$ bias of the energy and He density distribution for the $\mathrm{Rb}^{*} \mathrm{He}_{2}$ exciplex, which is small enough to investigate the bias down to very small $\Delta \tau$, hence large $M$.

\section{RESULTS FOR $\mathbf{R b}^{\star} \mathrm{He}_{n}$ CLUSTERS}

In this section, we focus on helium clusters doped with one $\mathrm{Rb}$ atom excited in the second excited electronic state. Both DMC and PIMC studies are presented after an analysis of the nontrivial $1^{2} \Pi_{3 / 2}$ potential energy surface of $\mathrm{Rb}^{*} \mathrm{He}_{n}$ cluster.

\section{A. Potential surface}

The shape of the potential surfaces for $\mathrm{Rb}^{*} \mathrm{He}_{n}$, obtained by diagonalization of the $6 \times 6$ matrix of the DIM formalism, ${ }^{30,32}$ is not trivial. The diagonalization of this matrix invalidates the usual conclusions drawn from a sumof-pairs for potential approximation. The potential energy surfaces for the two excited electronic states of $\mathrm{Rb}^{*} \mathrm{He}_{n}$ clusters considered in paper $\mathrm{I}$ and in this work, namely ${ }^{2} \Pi_{1 / 2}$ and ${ }^{2} \Pi_{3 / 2}$, are very different. The differences have already been underlined by thermodynamic considerations. ${ }^{17}$ Some understanding of the potential can be gained from planar cuts of the potential for small cluster sizes. For example, the one-dimensional $\mathrm{Rb}^{*} \mathrm{He}^{2} \Pi_{1 / 2}$ curve presents both a local (metastable) minimum and a global minimum separated by a barrier of $32.7 \mathrm{~cm}^{-1}$. In contrast, the one-dimensional $\mathrm{Rb}^{*} \mathrm{He}$ ${ }^{2} \Pi_{3 / 2}$ curve presents a single minimum with no barrier for an approaching helium atom. When using the $a b$ initio potential curves of Pascale, ${ }^{35,36}$ the one-dimensional potential well supports five vibrational states, which is in reasonable agreement with the calculation of Hirano et al. ${ }^{11}$ based on a more recent ${ }^{2} \Pi_{3 / 2}$ ab initio curve for which six vibrational levels are found.

Figure 1 presents on the top panel a $2 \mathrm{D}$ cut of the $3 \mathrm{D}$ potential surface of $\mathrm{Rb}^{*} \mathrm{He}_{2}$. More precisely, the potential surface is explored by moving a $\mathrm{He}$ atom around a rigid $\mathrm{Rb}^{*} \mathrm{He}_{1}$ exciplex. In this plot, $\mathrm{Rb}^{*}$ is located at the origin while one helium atom is fixed at coordinates $(6.25$, 0) $a_{0}$ which corresponds to the minimum of the $\mathrm{Rb}^{*} \mathrm{He}_{1}$ potential. The global potential minimum is found for a linear geometry $\mathrm{He}-\mathrm{Rb}^{*}-\mathrm{He}$ with an energy of $-186 \mathrm{~cm}^{-1}$. The second helium atom does not need to overcome a potential barrier to reach this minimum. However, the corresponding angle of approach is a quite small cone opened opposite to the direction to the first He atom. A local minimum 

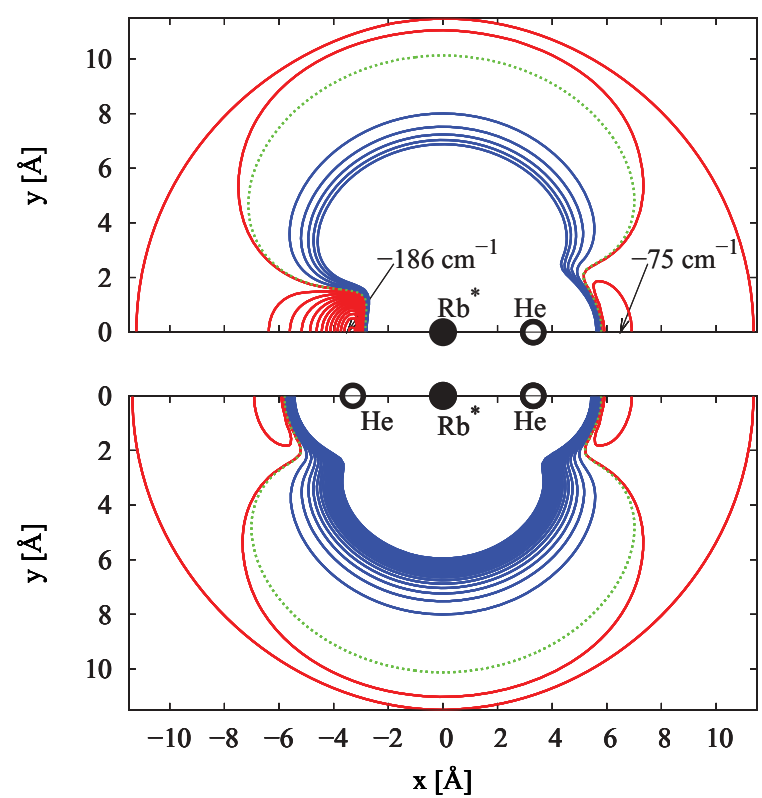

FIG. 1. (Top panel) Equipotential contour lines of the potential energy of $\mathrm{Rb}^{*} \mathrm{He}_{2}$ with $\mathrm{Rb}$ at the origin and a helium atom fixed at $(6.35,0) a_{0}$. The red and blue equipotential contours are in steps of $10 \mathrm{~cm}^{-1}$, where blue/red corresponds to repulsive/attractive interaction with respect to the asymptotic potential value (green contour) when the moving $\mathrm{He}$ atom reaches infinite distances. (Bottom panel) Same for $\mathrm{Rb}^{*} \mathrm{He}_{3}$ with the second helium fixed at $(-6.35,0) a_{0}$.

is found for a linear geometry $\mathrm{Rb}^{*}-\mathrm{He}-\mathrm{He}$ with an energy of $-75 \mathrm{~cm}^{-1}$. As underlined by the red contour lines, we note that there is a tiny shallow attractive part of the potential surface all around $\mathrm{Rb}^{*} \mathrm{He}_{1}$. This attractive part is however less than $1 \mathrm{~cm}^{-1}$ below the asymptotic value for $\mathrm{Rb}^{*} \mathrm{He}_{1}+\mathrm{He}$ (green contour line in Fig. 1). The shape of this potential is compatible with the formula given by Dupont-Roc, ${ }^{17}$

$$
V_{\sigma}^{5 p}(R) \sin ^{2} \theta+V_{\pi}^{5 p}(R)\left(1+\cos ^{2} \theta\right),
$$

obtained by the minimization of thermodynamical potentials. The lower panel of Fig. 1 illustrates that a third and subsequent helium atoms cannot bind strongly to $\mathrm{Rb}^{*}$ since the two most favorable positions are already taken by two He atoms. In this plot, a second helium atom is fixed at $(-6.25,0) a_{0}$ while a third helium atom is moved in the $(x, y)$ plane. As evidenced by the attractive contour lines, one can see that the third helium atom is much less attracted to the $\mathrm{Rb}^{*}$ atom than the first two. Therefore the cluster can be rationalized as a core exciplex $\mathrm{He}-\mathrm{Rb}^{*}-\mathrm{He}$ with additional $\mathrm{He}$ atoms bound weakly. This classical analysis of the potential energy surface of the ${ }^{2} \Pi_{3 / 2}$ state of $\mathrm{Rb}$ indicates that exciplexes with $n>2$ will not be formed in agreement with the work of Hofer et al. ${ }^{19}$ in which it is shown that only the excitation to the $\Pi_{1 / 2}$ state leads to formation of exciplexes with $n>2$ in solid He.

While for the second helium atom, the inspection of the potential surface alone (see Fig. 1) clearly indicates that the symmetric $\mathrm{He}-\mathrm{Rb}^{*}-\mathrm{He}$ cluster is more favorable than the asymmetric $\mathrm{Rb}^{*}-\mathrm{He}_{2}$ geometry, the conclusion for the larger clusters is not a priori known. Given the weak HeHe interaction and the restricted geometry where the interaction between the core exciplex $\mathrm{He}-\mathrm{Rb}^{*}-\mathrm{He}$ and additional $\mathrm{He}$ atoms is attractive, it is not obvious whether $\mathrm{Rb}^{*} \mathrm{He}_{n}$

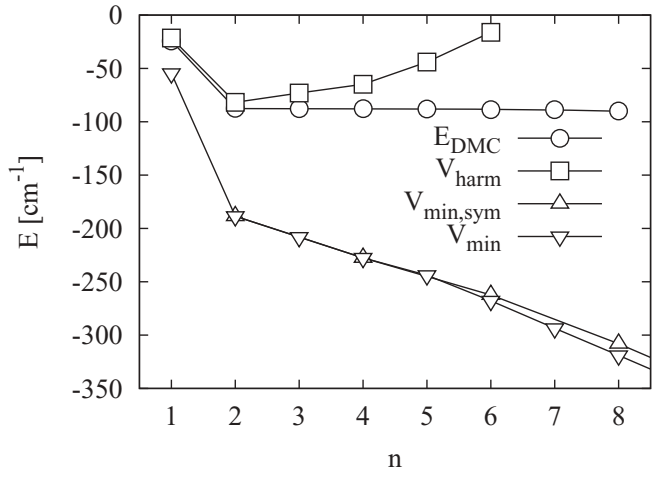

FIG. 2. Energies (in $\mathrm{cm}^{-1}$ ) as a function of $n$, number of helium atoms in the cluster. The global potential minimum $(\nabla)$, the minimum for "symmetric" clusters $(\triangle)$, the zero point energies within the harmonic approximation $(\square)$, and the quantum mechanical zero-point energies $(\bigcirc)$ are shown. For these last ones, the error bars of the DMC calculation are smaller than the symbol size.

clusters will have a symmetric or asymmetric structure. Symmetric $\mathrm{Rb}^{*} \mathrm{He}_{n}$ clusters, where $\mathrm{He}$ atoms are distributed evenly on both ends of the linear core exciplex $\mathrm{He}-\mathrm{Rb}^{*}-\mathrm{He}$, are preferred if the attraction to $\mathrm{Rb}^{*}$ wins against the $\mathrm{He}-\mathrm{He}$ attraction. Asymmetric clusters, where the additional $\mathrm{He}$ atoms aggregate on one end of $\mathrm{He}-\mathrm{Rb}^{*}-\mathrm{He}$, are preferred if the cumulative $\mathrm{He}-\mathrm{He}$ attractions win. ${ }^{49}$

In Fig. 2, the global minimum of potential as well as the minimum imposing a $\mathrm{He}_{n / 2}-\mathrm{Rb}^{*}-\mathrm{He}_{n / 2}$ symmetric structure are presented. We note that, at this classical level, the asymmetrical conformations are more stable than the symmetric conformations: the minimum of potential for $\mathrm{Rb}^{*} \mathrm{He}_{4}$ is symmetric, while for $n=6$ and $n=8$ it corresponds to asymmetric geometries. For the largest cluster presented in the figure, $\mathrm{Rb}^{*} \mathrm{He}_{8}$, the symmetric local minimum $\left(\mathrm{He}_{4}-\mathrm{Rb}^{*}-\mathrm{He}_{4}\right)$ is $10 \mathrm{~cm}^{-1}$ above the global minimum $\mathrm{He}_{1}-\mathrm{Rb}^{*}-\mathrm{He}_{7}$. A normal mode analysis has been performed for these (asymmetric) minimum geometries. The corresponding ground state energies are shown in Fig. 2. When comparing with exact DMC results (see below), the harmonic approximation is rather good for $n=1$ and $n=2 \mathrm{He}$ atoms, but fails completely for higher $n$. This is expected, given the strong anharmonicity of the potential surface. In the harmonic approximation, the $n>3$ clusters are not even bound.

\section{B. Energetics and symmetry}

The exact vibrational ground state of $\mathrm{Rb}^{*} \mathrm{He}_{n}$ clusters at $T=0 \mathrm{~K}$ is calculated using the second order IS-DMC method described in Sec. II B, and the results are reported in Fig. 2 and in Table I. Depending on cluster size, number of walkers, and desired error bars the imaginary time evolution $\tau$ after equilibration was chosen between $10^{7}$ and $10^{10}$ atomic units for each imaginary time step $\Delta \tau$ and each ensemble size $N_{\text {wlk. }}$. Successive quadratic extrapolations to $\Delta \tau \rightarrow 0$ and linear extrapolations $1 / N_{\mathrm{wlk}} \rightarrow 0$ are performed to overcome the time step bias and ensemble size bias. For all clusters studied, it was found that convergence of simulations with respect to time step, ensemble size and equilibration of the ensemble is rather slow. We assume that it is because in the 
TABLE I. Vibrational ground state energies computed using the IS-DMC method as a function of $n$ for $\mathrm{Rb}^{*} \mathrm{He}_{n}\left[E_{0}(n)\right]$ clusters. Corresponding chemical potentials $\mu(n)$ are also given. All energies are given in $\mathrm{cm}^{-1}$.

\begin{tabular}{lcc}
\hline \hline$n$ & $E_{0}(n)$ & $\mu(n)$ \\
\hline 1 & $-24.25 \pm 0.001$ & $-24.25 \pm 0.001$ \\
2 & $-87.64 \pm 0.004$ & $-63.39 \pm 0.005$ \\
3 & $-87.67 \pm 0.002$ & $-0.03 \pm 0.006$ \\
4 & $-87.77 \pm 0.01$ & $-0.09 \pm 0.012$ \\
5 & $-88.13 \pm 0.05$ & $-0.36 \pm 0.06$ \\
6 & $-88.42 \pm 0.04$ & $-0.30 \pm 0.09$ \\
7 & $-88.81 \pm 0.08$ & $-0.39 \pm 0.12$ \\
8 & $-89.84 \pm 0.19$ & $-1.03 \pm 0.27$ \\
\hline \hline
\end{tabular}

DMC simulation $\mathrm{He}$ atoms have a finite probability to wander from one end of $\mathrm{He}-\mathrm{Rb}^{*}-\mathrm{He}$ exciplex to the other. In the case of the $\mathrm{Rb}^{*} \mathrm{He}$ dimer, the convergence of our DMC simulation is checked by comparison to the result obtained by a standard basis set method. The corresponding energies are $-24.250 \pm 0.001 \mathrm{~cm}^{-1}$ for DMC and $-24.248 \mathrm{~cm}^{-1}$ when using basis set expansion.

The exact energies of small clusters with $n \leq 2$ almost coincide with the harmonic approximation values, but for larger clusters, the harmonic approximation becomes very poor, as seen in Fig. 2. As observed for the first electronic state (see paper I), the potential energy minimum explored during the simulation corresponds to the true global minimum only for small clusters $n \leq 4$. For larger ones, it deviates, indicating a negligible weight of the corresponding geometry in the vibrational ground state wavefunction.

In the discussion of the $\mathrm{Rb}^{*}-\mathrm{He}_{n}$ potential energy surface we concluded that $\mathrm{Rb}^{*} \mathrm{He}_{n}$ clusters should consist of a linear core exciplex $\mathrm{He}-\mathrm{Rb}^{*}-\mathrm{He}$ and $n-2$ much weaker bound $\mathrm{He}$ atoms. Inspection of the energies and the chemical potential $\mu(n)=E_{0}(n)-E_{0}(n-1)$ confirms that $\mathrm{Rb}^{*} \mathrm{He}_{n}$ contains two deeply bound helium atoms: while $\mu(n)$ is large for $n \leq 2$, the chemical potential $\mu(n)$ is much smaller for $n>2$, with only a slight systematic increase in magnitude as $n$ increases (see Table I). However, given the overall small magnitude of $\mu$, one in fact has to ask if $\mathrm{Rb}^{*} \mathrm{He}_{n}$ is bound at all or if a pure $\mathrm{He}_{n-2}$ cluster separated from the $\mathrm{Rb}^{*} \mathrm{He}_{2}$ core exciplex is the real ground state. The comparison of the energies presented in Table I to the sum of $\mathrm{Rb}^{*} \mathrm{He}_{2}$ cluster plus the one of a pure $\mathrm{He}_{n-2}$ cluster, leads to the conclusion that $\mathrm{Rb}^{*} \mathrm{He}_{n}$ is indeed stable as seen below.

It was shown that for an even number of helium atoms, the global minimum of the potential energy surface is attained for asymmetric configurations, where He atoms aggregate on one end of the core $\mathrm{He}-\mathrm{Rb}^{*}-\mathrm{He}$ exciplex. However, inspection of the multidimensional potential surface neglects zero-point motion of $\mathrm{He}$ atoms. The failure of the harmonic approximation indicates that effects of zero point motion are substantial, as expected for superfluid helium. Thus, we investigate whether the true quantum mechanical ground state of $\mathrm{Rb}^{*} \mathrm{He}_{n}$, with $n \geq 4$, corresponds to a symmetric or asymmetric distribution of He atoms. Furthermore, we investigate whether we can assign an approximate energy difference between the symmetric and asymmetric clusters, which would be possible for slow tunneling from one end of $\mathrm{He}-\mathrm{Rb}^{*}-\mathrm{He}$ to the other.

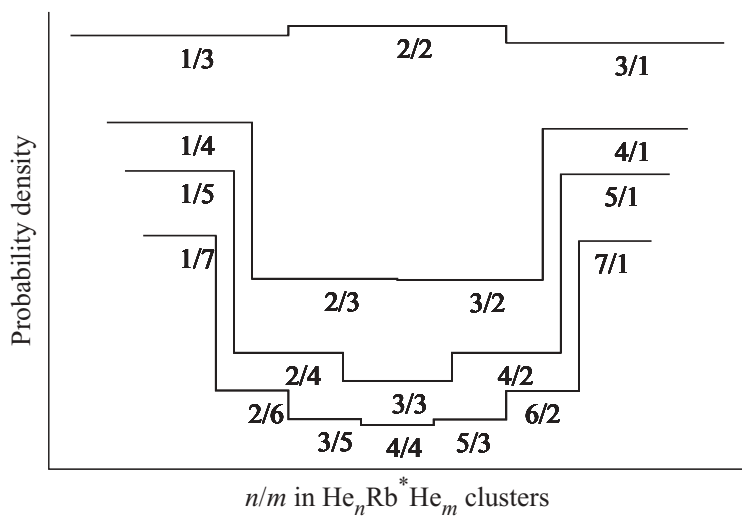

FIG. 3. Symmetry distribution of $\mathrm{He}_{n}-\mathrm{Rb}^{*}-\mathrm{He}_{m}$ clusters with 4,5 , 6, and 8 heliums in arbitrary units. The curves give the number of helium atoms on each side of the repulsive plane of the $1^{2} \Pi_{3 / 2}$ interaction with the $\mathrm{He}-\mathrm{Rb}{ }^{*}$ -He core exciplex. The small error bars are left out for clarity reason.

In order to measure the asymmetry of the clusters in $\mathrm{DMC}$, we determine the number of weakly bound $\mathrm{He}$ atoms on both sides of the repulsive plane of the $1^{2} \Pi_{3 / 2}$ interaction with the $\mathrm{He}-\mathrm{Rb}^{*}-\mathrm{He}$ core exciplex. In practice, we calculate the sign of the dot product, $\alpha_{n}^{i}=\operatorname{sgn}\left(\mathbf{r}_{\min }^{i} \cdot \mathbf{r}_{n}^{i}\right)$, for each $\mathrm{He}$ atom $n$ and each walker $i$. Here $\mathbf{r}_{\min }^{i}$ or $\mathbf{r}_{n}^{i}$ is the vector between $\mathrm{Rb}^{*}$ and the He closest to $\mathrm{Rb}^{*}$ or the other He atoms, respectively. $\alpha=\sum_{i, n} \alpha_{n}^{i}$, the number of positive versus negative $\alpha_{n}^{i}$, is a measure of symmetry. Figure 3 shows a histogram of $\alpha$, i.e., the probability of the symmetry distribution. It reveals that for clusters $\mathrm{Rb}^{*} \mathrm{He}_{n}$ with $n=4$ all possible structures have equal probability. That is, for $n=4 \mathrm{He}$ atoms, the conformation might be symmetric (two He atoms on each side) or asymmetric ( 1 versus $3 \mathrm{He}$ ). However, as the number $n$ of He atoms increases, the asymmetric structure, such that there is only one $\mathrm{He}$ (part of the $\mathrm{He}-\mathrm{Rb}^{*}-\mathrm{He}$ exciplex) on one side and the other He atoms aggregating on the other side, becomes more probable. Still, the probability to achieve a symmetric conformation is non-negligible. Since $\alpha$ was obtained in the mixed estimator approximation, Fig. 3 only serves to illustrate the trend toward asymmetry, the exact distribution will be more peaked. We note that our DMC simulations ergodically sample all conformations. Presumably the "tunneling" of $\mathrm{He}$ atoms from one side to the other happens via evaporation of a weakly bound $\mathrm{He}$ atom that is then driven back toward the other $\mathrm{He}$ atoms by the guiding wave function, see Eq. (6). As discussed below, such tunneling is usually not observed in our PIMC simulations.

A possible explanation of these results can be derived from energetics of pure helium clusters. For pure helium clusters the chemical potential of adding helium atoms decreases monotonously with cluster size ${ }^{50,51}$ Hence, a $\mathrm{He}_{6}$ cluster is much more stable than two $\mathrm{He}_{3}$ clusters. $\mathrm{A} \mathrm{Rb}^{*} \mathrm{He}_{8}$ cluster consists of two core and six weakly bound $\mathrm{He}$ atoms. Assuming that the $\mathrm{Rb}^{\text {* }}$ perturbs only slightly the potential seen by the six outer $\mathrm{He}$ atoms, $\mathrm{a} \mathrm{He}_{7}-\mathrm{Rb}^{*}-\mathrm{He}$ should thus be more stable than $\mathrm{He}_{4}-\mathrm{Rb}^{*}-\mathrm{He}_{4}$. Since this argument neglects the $\mathrm{Rb}^{*}$ interaction with $\mathrm{He}$, our QMC (quantum Monte Carlo) simulations are needed for a definite answer to the question about cluster symmetry. 
Further confirmation of the preference for asymmetric $\mathrm{Rb}^{*} \mathrm{He}_{n}$ clusters comes from PIMC simulations, at finite temperatures but without possible bias from a guiding wave function. Table I presents PIMC results for the energies of small clusters of size $n=2,4,6$. We chose a low temperature of $T=0.078 \mathrm{~K}$ to avoid thermal evaporation as much as possible, and two different time steps, $\Delta \tau=(40 \mathrm{~K})^{-1}$ and $\Delta \tau=(80 \mathrm{~K})^{-1}$, for estimating the time step bias. The notation $n_{l} / n_{r}$ indicates the number of ${ }^{4} \mathrm{He}$ atoms on each side of the repulsive plane of the $1^{2} \Pi_{3 / 2}$ interaction with the $\mathrm{He}-\mathrm{Rb}^{*}-\mathrm{He}$ core exciplex (see Fig. 1). In almost all our PIMC simulations, we found that the initial configuration $\mathrm{He}_{n_{l}}-\mathrm{Rb}^{*}-\mathrm{He}_{n_{r}}$ is preserved, i.e., the tunneling probability or thermal activation of $\mathrm{He}$ atoms switching sides is too low to be observed within the time of a PIMC simulation. Thus, we are able to study $\mathrm{He}_{n_{l}}-\mathrm{Rb}^{*}-\mathrm{He}_{n_{r}}$ clusters for different $n_{l} / n_{r}$ combinations separately.

As discussed above, the first two ${ }^{4} \mathrm{He}$ atoms are strongly bound, forming a $\mathrm{Rb}^{*}-\mathrm{He}$ and a $\mathrm{He}-\mathrm{Rb}^{*}-\mathrm{He}$ exciplex, respectively. For $n=4$, the $2 / 2$ configuration appears to be slightly more stable than the asymmetric $3 / 1$ configuration, consistent with DMC simulations, see Fig. 3. However, we note that the error bars of the PIMC energies would have to be reduced significantly to account for such small differences unambiguously. The tendency of the $n=4$ cluster toward a symmetric configuration changes with increasing $n$. For $n=6$ the attraction among ${ }^{4} \mathrm{He}$ atoms wins over the weak $\mathrm{He}-\mathrm{Rb}^{*}$ attraction for $\mathrm{He}$ atoms beyond the $\mathrm{He}-\mathrm{Rb}^{*}-\mathrm{He}$ core. For $n=6$ the asymmetric configuration is the stable one, as the energy of the $5 / 1$ configuration is slightly lower than the $3 / 3$ configuration ( Table I). As for $n=4$, the large error bars do not permit a definite conclusion but this interpretation is again consistent with the DMC symmetry distribution in Fig. 3.

The energy differences between bound and dissociated states, i.e., binding energies, are more informative than absolute energies. We define the difference between the energy $E_{n}$ of $\mathrm{Rb}^{*} \mathrm{He}_{n}$ and the sum of the energy $E_{2}$ of the $\mathrm{He}-\mathrm{Rb}^{*}-\mathrm{He}$ core and the energy, $E_{n-2}^{\text {pure }}$, of the pure cluster $\mathrm{He}_{n-2}$ of the remaining atoms

$$
\Delta E_{2}=E_{n}-\left(E_{2}+E_{n-2}^{\text {pure }}\right) .
$$

The last two columns of Table II give $\Delta E_{2}$ for the two time steps $\Delta \tau=(40 \mathrm{~K})^{-1}$ and $\Delta \tau=(80 \mathrm{~K})^{-1}$. A negative value of $\Delta E_{2}$ means that $\mathrm{Rb}^{*} \mathrm{He}_{n}$ is stable with respect to removing the noncore $\mathrm{He}$ atoms. In case of $n=4$, the energy $E_{2}^{\text {pure }}$ of $\mathrm{He}_{2}$ is given by the thermal energy of two free particles,
$2 \frac{3}{2} k_{B} T=0.23 \mathrm{~K}$, since $\mathrm{He}_{2}$ is not bound at $T=0.078 \mathrm{~K}$ (the binding energy is less than $2 \mathrm{mK}$ ), ${ }^{52}$ while for $n=6$, the pure $\mathrm{He}_{4}$ cluster is bound. We obtained $E_{4}^{\text {pure }}$ by a separate PIMC simulation: $E_{4}^{\text {pure }}=-0.23 \mathrm{~cm}^{-1} \pm 0.02 \mathrm{~cm}^{-1}$, $\left(-0.22 \mathrm{~cm}^{-1} \pm 0.02 \mathrm{~cm}^{-1}\right)$ for $\Delta \tau=(40 \mathrm{~K})^{-1}(\Delta \tau$ $\left.=(80 \mathrm{~K})^{-1}\right)$. As expected, the pair density approximation for the density matrix of a pure $\mathrm{He}$ cluster yields results that are already converged with a time step of $\Delta \tau=(40 \mathrm{~K})^{-1}$. From $\Delta E_{2}$ we see that for $n=4$ the symmetric configuration is more strongly bound while for $n=6$, it is already the asymmetric configuration that is more strongly bound.

As discussed in the appendix, $\Delta \tau=(80 \mathrm{~K})^{-1}$ still leads to a strong bias of the total energies (and less so for the densities) that are therefore not reliable. However, for the energy differences $\Delta E_{2}, \Delta \tau=(80 \mathrm{~K})^{-1}$ and even $\Delta \tau=(40 \mathrm{~K})^{-1}$ is sufficient, provided that the exciplex core of the clusters stays the same. Table II demonstrates that the time step bias of $\Delta E_{2}$ is smaller than the statistical error. This is because the time step bias is primarily due to the strongly bound ${ }^{4} \mathrm{He}$ atoms while for weakly bound ${ }^{4} \mathrm{He}$ atoms the energy can be calculated reliably for $\Delta \tau=(40 \mathrm{~K})^{-1}$ or $\Delta \tau=(80 \mathrm{~K})^{-1}$.

For large clusters, the long path integral chains necessary for a low temperature of $T=0.078 \mathrm{~K}$ would render the simulations very inefficient. Furthermore, these clusters are more strongly bound so that we can perform simulations at a higher temperature without destroying the cluster by evaporation. Therefore, we perform large cluster simulations at a temperature of $T=0.31 \mathrm{~K}$. For a $\mathrm{Rb}^{*} \mathrm{He}_{n}$ cluster with $n=100$, we show results for the $\mathrm{Rb}^{*} \mathrm{He}_{1}$ exciplex adsorbed on a cluster formed by the remaining $99 \mathrm{He}$ atoms, i.e., in the above notation, $n_{l}=100$ and $n_{r}=0$. This corresponds to the experimental observation that $\mathrm{Rb}^{*} \mathrm{He}$, i.e., an exciplex with a single $\mathrm{He}$ atom, rather than $\mathrm{He}-\mathrm{Rb}^{*}-\mathrm{He}$ forms preferentially upon electronic excitation. Unlike in experiment, where the exciplex can desorb due to the release of vibrational binding energy, in a simulation we can study the equilibrium configuration for an adsorbed $\mathrm{Rb}^{*} \mathrm{He}_{1}$ exciplex. Table III presents the energies for two different time steps and, in analogy to $\Delta E_{2}$, Eq. (11), the energy difference $\Delta E_{1}$ given by $\Delta E_{1}=E_{n}-\left(E_{1}+E_{n-1}^{\text {pure }}\right)$. The computed negative value of $\Delta E_{1}$ means that $\mathrm{Rb}^{*} \mathrm{He}$ forms a stable surface state on $\mathrm{He}_{99}$.

In Fig. 4 we show the ${ }^{4} \mathrm{He}$ density with respect to the $\mathrm{Rb}^{*}$ atom (the vertical axis is defined as intersecting $\mathrm{Rb}^{*}$ and the center of mass of $\mathrm{He}_{n}$ ). The sharp peak below the $\mathrm{Rb}^{*}$ atom (indicated by a green disk) is the $\mathrm{He}$ atom of the $\mathrm{Rb}^{*} \mathrm{He}$ exciplex. Note the cluster of the remaining $99 \mathrm{He}$ atoms is

TABLE II. Vibrational ground state energies obtained by PIMC $(T=0.078 \mathrm{~K})$ with two different time steps $\Delta \tau$. The last two columns give energy differences $\Delta E_{2}$ defined in the text. Energies are given in $\mathrm{cm}^{-1}$.

\begin{tabular}{cccccc}
\hline \hline$n$ & $n_{l} / n_{r}$ & $E_{n}{ }^{\mathrm{a}}$ & $E_{n}{ }^{\mathrm{b}}$ & $\Delta E_{2}{ }^{\mathrm{a}}$ & $\Delta E_{2}{ }^{\mathrm{b}}$ \\
\hline 2 & $1 / 1$ & $-113.7 \pm 0.1$ & $-97.5 \pm 0.1$ & & \\
4 & $2 / 2$ & $-113.8 \pm 0.1$ & $-97.8 \pm 0.1$ & $-0.3 \pm 0.2$ & $-0.4 \pm 0.2$ \\
4 & $3 / 1$ & $-113.7 \pm 0.1$ & $-97.7 \pm 0.1$ & $-0.2 \pm 0.2$ & $-0.3 \pm 0.2$ \\
6 & $3 / 3$ & $-114.4 \pm 0.1$ & $-98.2 \pm 0.1$ & $-0.6 \pm 0.2$ & $-0.5 \pm 0.2$ \\
6 & $5 / 1$ & $-114.7 \pm 0.1$ & $-98.6 \pm 0.1$ & $-0.8 \pm 0.3$ & $-0.8 \pm 0.2$ \\
\hline
\end{tabular}

${ }^{\mathrm{a}}$ For time step $\Delta \tau=(40 \mathrm{~K})^{-1}$

${ }^{\mathrm{b}}$ For time step $\Delta \tau=(80 \mathrm{~K})^{-1}$ 
TABLE III. Vibrational ground state energies obtained by PIMC $(T=0.31 \mathrm{~K})$. The last two columns give energy differences $\Delta E_{1}$ which are discussed in the text. Energies are given in $\mathrm{cm}^{-1}$.

\begin{tabular}{lccccc}
\hline \hline$n$ & $n_{l} / n_{r}$ & $E_{n}{ }^{\mathrm{a}}$ & $E_{n}{ }^{\mathrm{b}}$ & $\Delta E_{1}{ }^{\mathrm{a}}$ & $\Delta E_{1}{ }^{\mathrm{b}}$ \\
\hline 1 & $1 / 0$ & $-33.5 \pm 0.1$ & $-28.0 \pm 0.1$ & - & - \\
100 & $100 / 0$ & $-263.6 \pm 0.3$ & $-248.8 \pm 0.3$ & $-4.4 \pm 0.6$ & $-4.2 \pm 0.6$ \\
\hline
\end{tabular}

${ }^{\mathrm{a}}$ For time step $\Delta \tau=(40 \mathrm{~K})^{-1}$

${ }^{\mathrm{b}}$ For time step $\Delta \tau=(80 \mathrm{~K})^{-1}$

almost spherical, with only a small perturbation close to the $\mathrm{He}$ atom of the $\mathrm{Rb}^{*} \mathrm{He}$ exciplex.

The $n=100$ cluster can be seen as a system intermediate between small clusters that have been described so far and large ${ }^{4} \mathrm{He}_{n}$ droplets with $n=\mathrm{O}\left(10^{3}-10^{4}\right)$ as produced in experiments. A QMC simulation of such large droplets would require significant computational resources. Instead, we present results for the limit of a flat ${ }^{4} \mathrm{He}$ surface in Sec. IV.

\section{RESULTS FOR Rb ON A ${ }^{4} \mathrm{He}$ SURFACE}

In several experiments, $,{ }^{9} 12,13,31 \mathrm{Rb}$ atoms attached to large ${ }^{4} \mathrm{He}$ droplets are excited from the electronic ground state to the $1^{2} \Pi_{3 / 2}$ state. Whereas there are still discrepancies concerning the interpretation of these experiments, especially concerning the dynamics of the process, there is agreement that upon excitation, a $\mathrm{Rb}^{*} \mathrm{He}$ exciplex is formed, which desorbs from the surface. Note that $\mathrm{Rb}^{*} \mathrm{He}_{2}$ exciplex formation has not been observed although for $\mathrm{Rb}$ in the $\Pi_{3 / 2}$ state it would be energetically favorable as we have seen in Sec. III. That lack of evidence for $\mathrm{Rb}^{*} \mathrm{He}_{2}$ exciplexes points to a low cross section for this process. A calculation of the cross sections for exciplex formation would require solving the time dependent $(n+1)$-body Schrödinger equation. Since this is numerically impossible for large $n$, we have to make approximations.

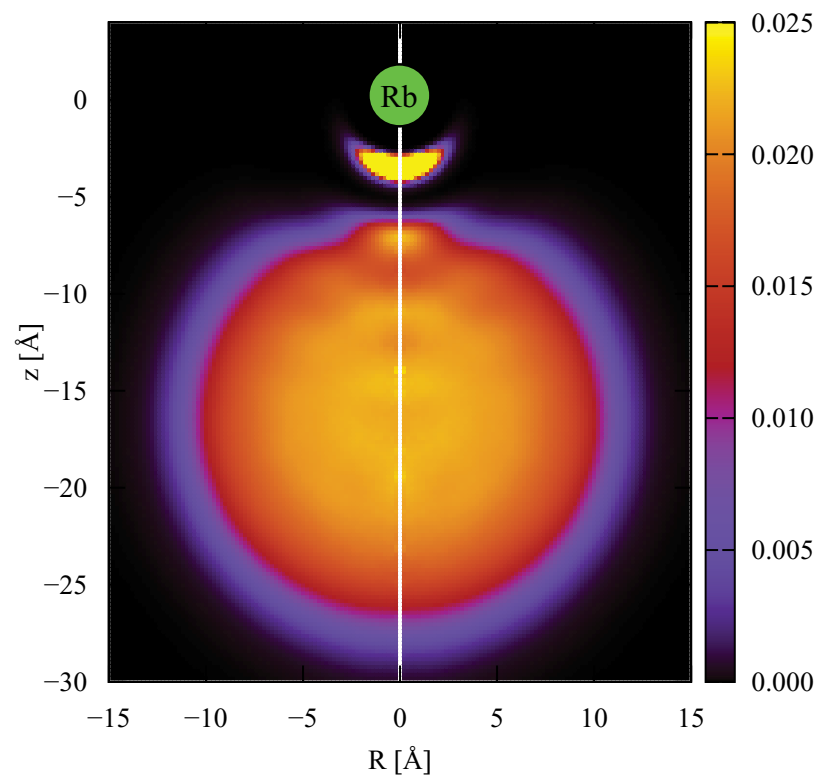

FIG. 4. Color map of the He density relative to $\mathrm{Rb}^{*}$ for $\mathrm{Rb}^{*} \mathrm{He}_{100}$ at $0.31 \mathrm{~K}$, obtained by PIMC with time step $\Delta \tau=(80 \mathrm{~K})^{-1}$. The position of $\mathrm{Rb}^{*}$ is marked by the green disk. The yellow spot corresponds to the He atom of the $\mathrm{Rb}^{*} \mathrm{He}$ exciplex.
Following the ideas of paper I, we perform a "vertical Monte Carlo transition," consisting of an abrupt switch of the PES from $\mathrm{Rb}$ to $\mathrm{Rb}^{*}$ in the course of a PIMC simulation. Thus we are able to see how $\mathrm{Rb}^{*} \mathrm{He}_{n}$ equilibrates starting from $\mathrm{RbHe}_{n}$ configurations. Since PIMC does not simulate the dynamics of the system, this is not a physical equilibration, but a Monte Carlo equilibration where the $\mathrm{Rb}$ adsorption state converges to the closest (meta-)stable $\mathrm{Rb}^{*}$ adsorption state (if there is one).

As in paper I, we turn to ${ }^{4} \mathrm{He}$ films to emulate the surface of large droplets of thousands of ${ }^{4} \mathrm{He}$, since the latter would be computationally too demanding. The chosen simulation size (a film consisting of $n=224 \mathrm{He}$ atoms) is not prohibitively large. However, we are interested here in the properties of a single $\mathrm{Rb}$ atom and its local environment, thus quantities of the order $1 / n$. In order to keep the computational effort feasible, we increase the temperature to about twice the experimentally determined temperature of ${ }^{4} \mathrm{He}$ droplets, namely $T=0.625 \mathrm{~K}$. The time step used, $\Delta \tau=(80 \mathrm{~K})^{-1}$, is justified along with the discussion of the results. ${ }^{53}$

As for the excitation to the $\Pi_{1 / 2}$ state in paper I, we observe that the "vertical MC transition" from the $\Sigma_{1 / 2}$ to the $\Pi_{3 / 2}$ state is followed by a relaxation to a surface state of $\mathrm{Rb}^{*}$. In Fig. 5, we show the density $\rho_{\mathrm{Rb}}(z)$ of the adsorbed $\mathrm{Rb}$ in

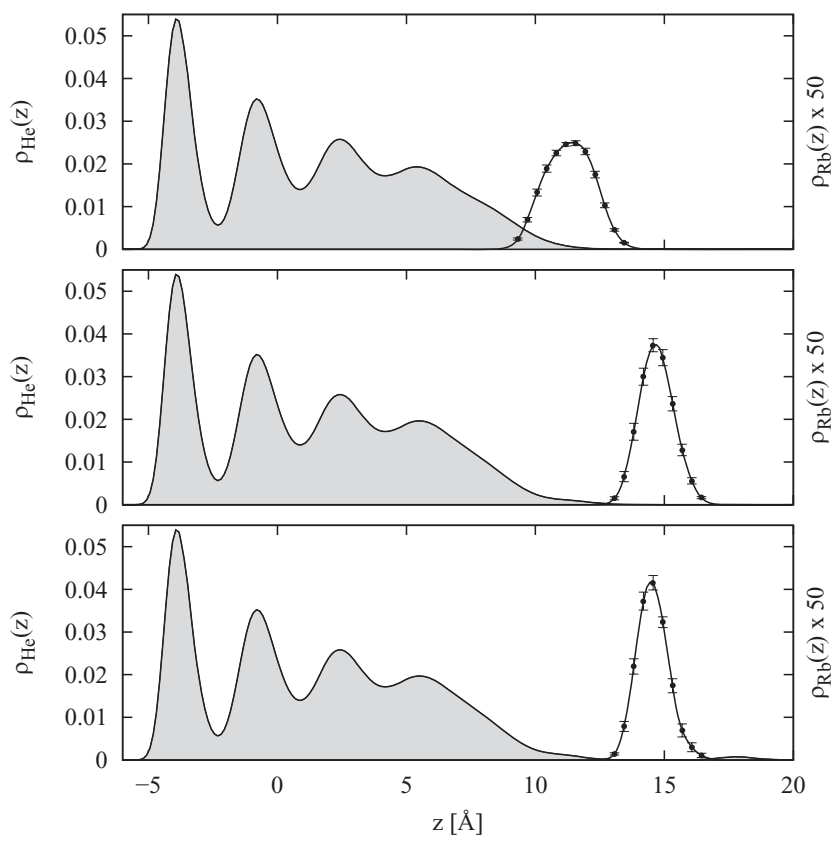

FIG. 5. The density profile $\rho_{\mathrm{He}}(z)$ of the ${ }^{4} \mathrm{He}$ film (shaded area) and the density $\rho_{\mathrm{Rb}}(z)$ of adsorbed $\mathrm{Rb}$ (top panel) and $\mathrm{Rb}^{*}$ (other two panels) are shown. The middle and bottom panels correspond to an adsorbed $\mathrm{Rb}^{*} \mathrm{He}_{1}$ and $\mathrm{HeRb}^{*} \mathrm{He}$ exciplex, respectively. Error bars of $\rho_{\mathrm{Rb}}(z)$ are shown for every third sampling bin, while error bars of $\rho_{\mathrm{He}}(z)$ are negligible. 


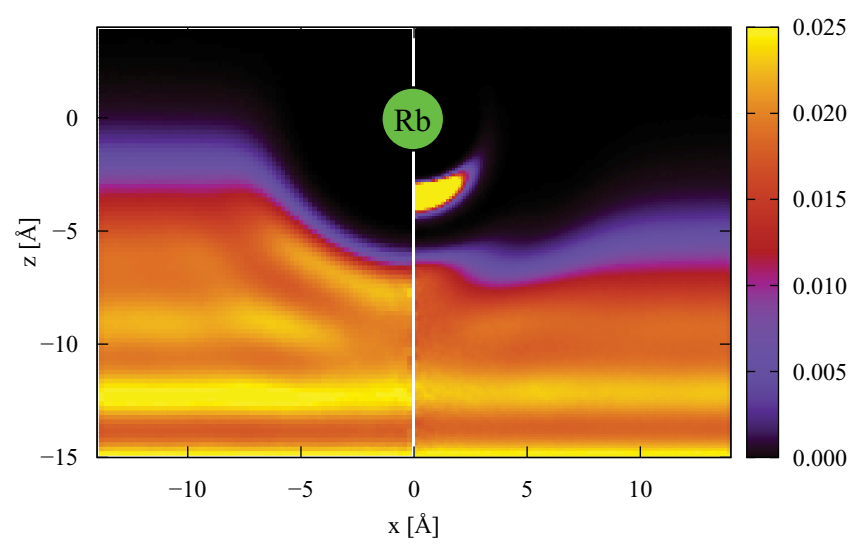

FIG. 6. Color map of the density distribution of the ${ }^{4} \mathrm{He}$ film with respect to an adsorbed $\mathrm{Rb}$ atom before (left half) and after (right half) the transition from the electronic ground state $\Sigma_{1 / 2}$ to the excited $\Pi_{3 / 2}$ state. The origin $(0,0)$ of coordinate system coincides with the position of $\mathrm{Rb} / \mathrm{Rb}{ }^{*}$, indicated by a green disk.

the electronic ground state $\Sigma_{1 / 2}$ in the top panel from which we initiate the vertical MC transition. After the transition, the $\mathrm{Rb}^{*}$ atom relaxes to the final density in the $\Pi_{3 / 2}$ state, which is shown in the middle panel. $\mathrm{Rb}^{*}$ is clearly located farther from the surface. The film density (shaded areas) is little affected by the transition which means that the simulated surface area is large enough to minimize finite size effects.

Inspection of only $\rho_{\mathrm{Rb}}(z)$ could lead to the conclusion that the result is the same as for the transition to $\Pi_{1 / 2}$ where no exciplex was found in paper I. However, closer inspection reveals a qualitatively new picture. The energy drops by $101 \mathrm{~cm}^{-1}$ after relaxation, which indicates the formation of an exciplex. The proof for the fact that this is indeed the case is provided by Fig. 6 where we show a vertical cut through the film, with the ${ }^{4} \mathrm{He}$ density given in false color representation relative to the position of $\mathrm{Rb} / \mathrm{Rb}^{*}$ (green disk), which is set to be at the origin in the figure. The left half of the color map corresponds to the ground state ${ }^{4} \mathrm{He}$ density relative to the $\mathrm{Rb}$ atom and the right half to the relaxed ${ }^{4} \mathrm{He}$ density after excitation of $\mathrm{Rb}$ to the $\Pi_{3 / 2}$ state. It is clear that $\mathrm{Rb}^{*}$ forms an exciplex with a single ${ }^{4} \mathrm{He}$ atom as illustrated by the high density below the Rb atom ("overexposed" yellow area). $\mathrm{Rb}^{*} \mathrm{He}$ is like an adsorbed molecule. Its orientation perpendicular to the surface is stabilized by the mostly repulsive ring, perpendicular to the $\mathrm{Rb}^{*} \mathrm{He}$ axis, in the interaction potential (see Fig. 1), i.e., parallel to the surface. The picture of $\mathrm{Rb}^{*} \mathrm{He}$ as a molecule adsorbed to a He surface is appropriate, since in our PIMC simulations we observe that the $\mathrm{He}$ atom bound to $\mathrm{Rb}^{*}$ never participates in Bose exchange with the other He atoms. Note that unlike for adsorbed $\mathrm{Rb}$ in the electronic ground state, there is basically no dimple in the $\mathrm{He}$ surface below the $\mathrm{Rb}^{*} \mathrm{He}$ exciplex.

The small increase of ${ }^{4} \mathrm{He}$ density below $\mathrm{Rb}{ }^{*} \mathrm{He}$ indicates an attractive funnel-shaped potential region. Although the $\Pi_{3 / 2}$ potential could accommodate a second ${ }^{4} \mathrm{He}$ atom on the opposite side (above the $\mathrm{Rb}$ atom in Fig. 6), none of our vertical MC transitions lead to such a $\mathrm{Rb}^{*} \mathrm{He}_{2}$ exciplex. For the formation of $\mathrm{Rb}^{*} \mathrm{He}_{2} \mathrm{a}^{4} \mathrm{He}$ atom would have to overcome both the chemical potential of the ${ }^{4} \mathrm{He}$ film $(-7.2 \mathrm{~K}$ $=5 \mathrm{~cm}^{-1}$ ) and the geometric barrier of the repulsive ring of
TABLE IV. Binding energy $\Delta E_{i}$ of a $\mathrm{Rb}$ atom and of exciplexes $\mathrm{Rb}{ }^{*} \mathrm{He}$ and $\mathrm{Rb}^{*} \mathrm{He}_{2}$ adsorbed on the surface of a ${ }^{4} \mathrm{He}$ film, at temperature $T=0.625 \mathrm{~K}$. The middle and the right columns provide the average distance $\langle z\rangle$ to the surface in $\AA$ (see text for definition) and the width $\Delta z$ of the density $\rho_{\mathrm{Rb}}(z)$ of the surface state of $\mathrm{Rb} / \mathrm{Rb}^{*}$, respectively.

\begin{tabular}{lrcc}
\hline \hline & $\Delta E_{i}\left[\mathrm{~cm}^{-1}\right]$ & $\langle z\rangle[\AA]$ & $\Delta z$ \\
\hline $\mathrm{Rb}$ & $-10.9 \pm 0.5$ & 3.3 & 0.84 \\
$\mathrm{Rb}^{*} \mathrm{He}$ & $-9.5 \pm 0.8$ & 6.7 & 0.45 \\
$\mathrm{Rb}^{*} \mathrm{He}_{2}$ & $-11.0 \pm 0.7$ & 6.6 & 0.39 \\
\hline \hline
\end{tabular}

the $\mathrm{Rb}^{*}-\mathrm{He}$ potential before it can find the second potential minimum. This energy barrier is too big for this "process" to happen during $\mathrm{MC}$ relaxation.

A vertical MC transition cannot make any quantitative statements about the dynamics of the $\mathrm{Rb}$ atom and the $\mathrm{He}$ film after excitation $1^{2} \Sigma_{1 / 2} \rightarrow 1^{2} \Pi_{3 / 2}$. But we expect that the above arguments still apply qualitatively for the dynamics that $\mathrm{Rb}^{*} \mathrm{He}_{1}$ exciplex formation is the most likely process, while $\mathrm{Rb}^{*} \mathrm{He}_{2}$ is either not formed or it is formed with low probability. What is lost in our MC transition is of course energy conservation. For $\Sigma_{1 / 2} \rightarrow \Pi_{1 / 2}$ in paper I this violation was less essential because no exciplex was formed, and the relaxation to a metastable adsorption state was in agreement with experiment. ${ }^{15}$ In the present situation, however, there is a significant amount of exciplex binding energy released. In the MC relaxation, this energy is simply dissipated, but in reality energy is conserved and the released binding energy has to flow somewhere. If we could account for energy conservation in MC simulations, we would probably find a swift desorption as in experiments. ${ }^{7,9,12}$ Here we cannot discuss the dynamics of the desorption process, but only the surface state of $\mathrm{Rb}^{*} \mathrm{He}$ on a ${ }^{4} \mathrm{He}$ film found after relaxation. In addition, we added another He atom by hand to the other potential minimum of the $\mathrm{Rb}^{*}-\mathrm{He}$ interaction in order to study adsorbed $\mathrm{Rb}^{*} \mathrm{He}_{2}$.

Table IV lists the binding energies $\Delta E_{2}$ and $\Delta E_{1}$, defined in Sec. III, of $\mathrm{Rb}^{*} \mathrm{He}_{2}$ and $\mathrm{Rb}^{*} \mathrm{He}$, respectively, as well as the binding energy $\Delta E_{0}$ of $\mathrm{Rb}$ in the electronic ground state $1^{2} \Sigma_{1 / 2}$, from which the transition to the $1^{2} \Pi_{3 / 2}$ state starts. As explained above, $\Delta E_{i}$ is the energy difference between the adsorbed state and the desorbed state of $\mathrm{Rb}, \mathrm{Rb}{ }^{*} \mathrm{He}$, and $\mathrm{Rb}^{*} \mathrm{He}_{2}$. As in the study of small $\mathrm{Rb}^{*} \mathrm{He}_{n}$ clusters in Sec. III, determination of $\Delta E_{i}$ is computationally inefficient, since it is the difference between two large numbers and is therefore afflicted with large relative error bars.

We see that the release of the binding energy of $\mathrm{Rb}^{*}$ and $\mathrm{He}\left(-106.6 \mathrm{~cm}^{-1}\right.$ for the present time step of $\left.\Delta \tau=(80 \mathrm{~K})^{-1}\right)$ can easily overcome the energy of $-9.5 \mathrm{~cm}^{-1}$ binding the $\mathrm{Rb}^{*} \mathrm{He}$ exciplex to the surface, consistent with the experimental interpretation that the exciplexes desorb after formation on the surface of He droplets. The average distance $\langle z\rangle$ of $\mathrm{Rb}$ and $\mathrm{Rb}^{*}$ from the He surface is also shown in Table IV, which we define as the location $z_{0}$ where the density profile has half the bulk equilibrium density, $n\left(z_{0}\right)=\frac{1}{2} 0.022 \AA^{-3}$ $\left(z_{0}=8.0 \AA\right)$. As seen in Figs. 5 and $6,\langle z\rangle$ increases upon excitation $\Sigma_{1 / 2} \rightarrow \Pi_{1 / 2}$ because of the He atom bound to $\mathrm{Rb}^{*}$ that sits between the alkali atom and the surface.

While adsorbed $\mathrm{Rb}^{*}$ exciplexes are not observed in typical helium nanodroplet spectroscopy due to desorption, 


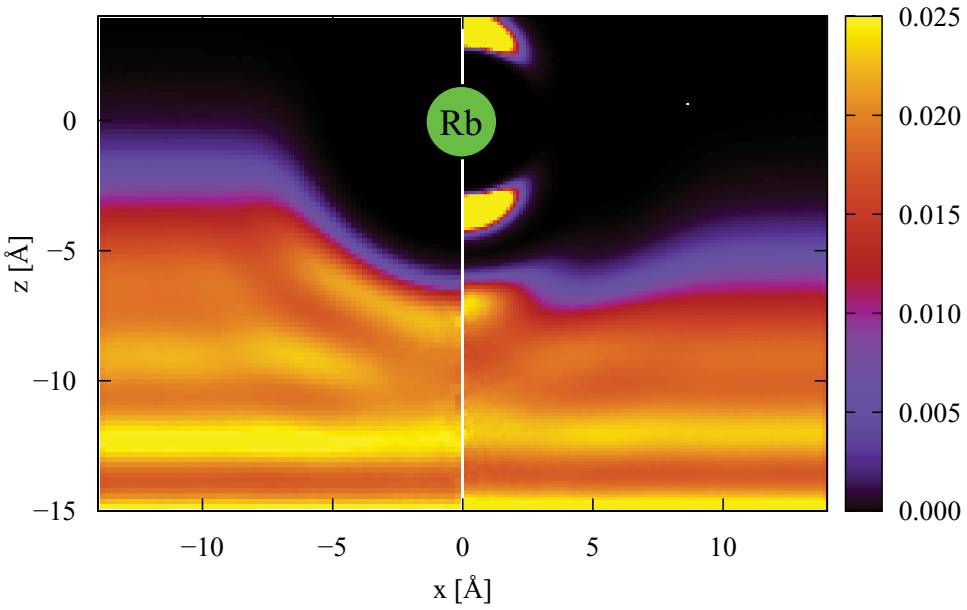

FIG. 7. Density distribution of ${ }^{4} \mathrm{He}$ film with respect to adsorbed $\mathrm{Rb}$ (left) and $\mathrm{Rb}^{*}$ (right) atom, which is located at $(0,0)$ (green disk).

we will not rule out that they might be observed in different experimental setups. For example, if exciplexes $\mathrm{Rb}^{*} \mathrm{He}$ are produced in ${ }^{4} \mathrm{He}$ gas such as in Ref. 11, they can be picked up by the droplet after production and be adsorbed in the surface state shown in Fig. 6. We therefore extended our study to adsorbed exciplexes with two $\mathrm{He}$ atoms and $\mathrm{He}-\mathrm{Rb}^{*}-\mathrm{He}$. The binding energy $\Delta E_{2}$ of $\mathrm{He}-\mathrm{Rb}^{*}-\mathrm{He}$ is given in Table IV, together with average distance $\langle z\rangle$ from the He surface and the width $\Delta z$ of the surface state. Much of the discussion of adsorbed $\mathrm{Rb}^{*} \mathrm{He}$ applies qualitatively also to adsorbed $\mathrm{He}-\mathrm{Rb}^{*}-\mathrm{He}$. Coincidentally, the binding energy $\Delta E_{1 / 2}$ of $\mathrm{Rb}^{*} \mathrm{He}$ and $\mathrm{He}-\mathrm{Rb}^{*}-\mathrm{He}$ are quite similar to that of $\mathrm{Rb}$.

The pickup of a $\mathrm{Rb}^{*} \mathrm{He}_{2}$ exciplex by a ${ }^{4} \mathrm{He}$ droplet would result in a structure shown in Fig. 7. As in Fig. 6, we show the ${ }^{4} \mathrm{He}$ density relative to $\mathrm{Rb}^{*}$ as color map. Compared to Fig. 6 there are now two yellow spots for the two He atoms of the $\mathrm{He}-\mathrm{Rb}^{*}-\mathrm{He}$ exciplex, one He between $\mathrm{Rb}^{*}$ and the surface, the other $\mathrm{He}$ on the opposite end. The tighter localization of the density of the $\mathrm{He}$ atom below $\mathrm{Rb}^{*}$ indicates less zeropoint motion. This is seen quantitatively in the last column of Table IV, where we compare the width $\Delta z \equiv\left\langle z^{2}\right\rangle-\langle z\rangle^{2}$ of the probability density for the Rb position in the $\Sigma_{1 / 2}$ ground state and the $\Pi_{3 / 2}$ state with one or two He atoms. Indeed, the motion of one of the two He atoms of $\mathrm{Rb}^{*} \mathrm{He}_{2}$ drags the spontaneous symmetry axis of the $\mathrm{Rb}^{*}-\mathrm{He}$ potential away from the other He atom which hence loses its favorable spot in the potential minimum. In other words, $\mathrm{Rb}^{*} \mathrm{He}_{2}$ behaves like a molecule, with a rotational constant $B$ of about twice the value of $B$ of $\mathrm{Rb}^{*} \mathrm{He}$, and accordingly about half the zero point motion. Like $\mathrm{Rb}^{*} \mathrm{He}$, the axis of the adsorbed $\mathrm{Rb}^{*} \mathrm{He}_{2}$ is perpendicular to the film, with a more pronounced density increase below $\mathrm{Rb}^{*} \mathrm{He}_{2}$, consistent with a slightly stronger binding to the surface, see Table IV.

\section{ELECTRONIC ABSORPTION SPECTRA}

The electronic absorption spectrum was semiclassically approximated by $\operatorname{Lax}^{33,54-56}$ to be proportional to

$S_{\mathrm{sc}}(E)=\left|M_{e g}\right|^{2} \int d \mathcal{X} p(\mathcal{X}) \delta\left(V_{e}(\mathcal{X})-V_{g}(\mathcal{X})-E\right)$, where $M_{e g}$ is the electronic transition dipole moment, assumed to be independent of the particle coordinates $\mathcal{X}$, and $V_{e}(\mathcal{X})$ and $V_{g}(\mathcal{X})$ are the total potential of the $\mathrm{Rb}-\mathrm{He}$ system for the excited and ground state of $\mathrm{Rb}$, respectively. Since the $\mathrm{He}-\mathrm{He}$ potential is assumed to be independent of the electronic state of $\mathrm{Rb}$, it cancels in the above expression. In PIMC, we simulate a system in the canonical ensemble at given finite temperature $T=\left(k_{B} \beta\right)^{-1}$ by sampling the diagonal $(n+1)$-body density matrix $\rho(\mathcal{X}, \mathcal{X}, \beta)$, see Eq. (9). Hence, in Eq. (12) we set $p(\mathcal{X})=\rho(\mathcal{X}, \mathcal{X}, \beta)$.

For a weakly bound cluster, like $\mathrm{RbHe}_{n}$, quantum effects are generally large, and the validity of approximation [Eq. (12)] that neglects kinetic energy has to be checked. Several improvements of the semiclassical approximation [Eq. (12)] have been proposed by Cheng and Whaley ${ }^{55}$ that incorporate the kinetic energy of the alkali-He cluster in the electronic ground state, but using the semiclassical approximation for the excited state. The predominant effect of these modifications of the Lax approximation was found to be a shift of the electronic spectrum. In Ref. 33, the electronic spectrum of $\mathrm{Li}, \mathrm{Na}$, and $\mathrm{K}$ on ${ }^{4} \mathrm{He}$ clusters was simulated with PIMC following one scheme by Cheng and Whaley where only the alkali kinetic energy is taken into account.

Our PIMC simulations at $0.31 \mathrm{~K}$ show that the kinetic energy $\left\langle T_{\mathrm{Rb}}\right\rangle$ of $\mathrm{Rb}$ in the electronic ground state slowly rises with the ${ }^{4} \mathrm{He}$ cluster size $n$, reaching about $\left\langle T_{\mathrm{Rb}}\right\rangle=1 \mathrm{~K}$ in the limit of $\mathrm{Rb}$ on the surface of a ${ }^{4} \mathrm{He}$ film. Correspondingly, the energy scale of the electronic absorption spectra is shifted by less than $1 \mathrm{~K}$. This correction is much smaller than the typical energies involved in the spectra such as the linewidths which are on the order of $100 \mathrm{~K}$ for large $n$. Therefore, we neglect the kinetic energy correction, considering that we have made other, presumably more severe approximations by using the DIM method for the excited potential surface $V_{e}$.

In Fig. 8, we show the PIMC results for the electronic absorption spectra from ground state $\Sigma_{1 / 2}$ to the excited states of $\mathrm{Rb}$ adsorbed to ${ }^{4} \mathrm{He}$ clusters of sizes $n=2$, 3, 20, 100, 200, 300, and to a ${ }^{4} \mathrm{He}$ film. The two vertical lines indicate the electronic transitions without ${ }^{4} \mathrm{He}$, the left vertical line corresponding to the $5^{2} \mathrm{~S}_{1 / 2} \rightarrow 5^{2} \mathrm{P}_{1 / 2}$ transition $\left(\mathrm{D}_{1}\right.$ line), and the right vertical line corresponding to the $5^{2}$ 


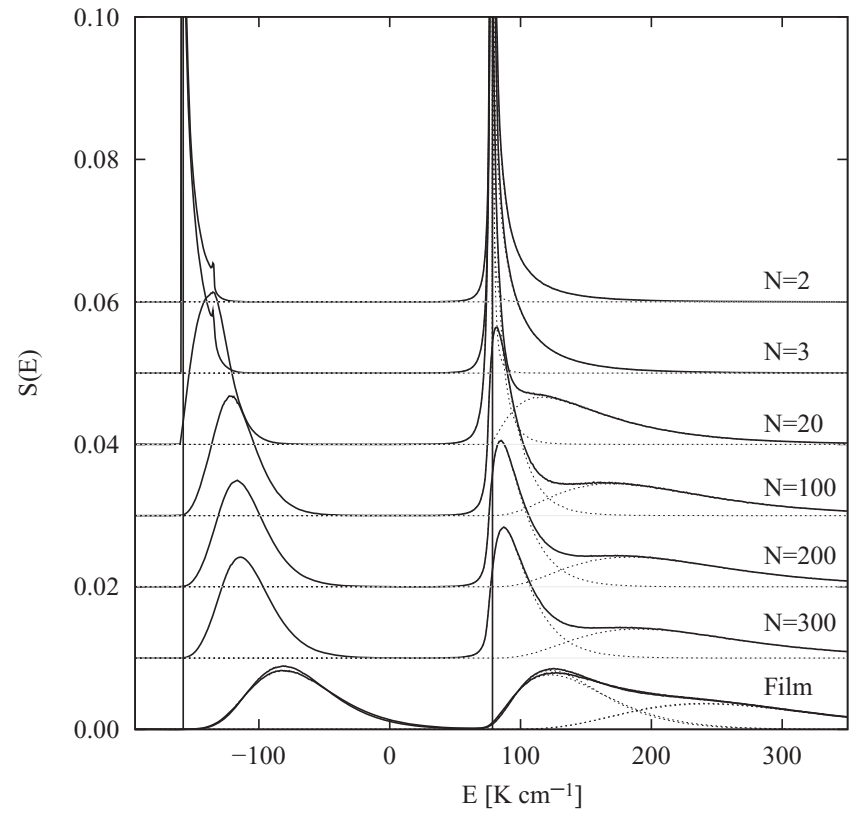

FIG. 8. Electronic absorption spectrum of $\mathrm{Rb}$ on ${ }^{4} \mathrm{He}$ clusters $(n=2,3,20,100,200,300)$ and on a ${ }^{4} \mathrm{He}$ film in the Lax approximation. For better visibility, the spectra are shifted downward with increasing $n$. The temperature is $T=0.31 \mathrm{~K}$. For the ${ }^{4} \mathrm{He}$ film, we also show the slightly broader spectrum at $T=0.62 \mathrm{~K}$ (lower curve).

$\mathrm{S}_{3 / 2} \rightarrow 5^{2} \mathrm{P}_{3 / 2}$ transition $\left(\mathrm{D}_{2}\right.$ line) for a free $\mathrm{Rb}$ atom. Both ${ }^{2} \Pi_{3 / 2}$ and ${ }^{2} \Sigma_{1 / 2}$ correlate to the $5^{2} \mathrm{P}_{3 / 2}$ degenerate state of the free $\mathrm{Rb}$ atom. We have separated the contributions to the $D_{2}$ transition from the ${ }^{2} \Pi_{3 / 2}$ and ${ }^{2} \Sigma_{1 / 2}$ state by showing each individual spectrum as dotted lines in Fig. 8 under the combined $D_{2}$ transition which is the sum of both. The temperature was set to $T=0.31 \mathrm{~K}$. For the ${ }^{4} \mathrm{He}$ film results, we investigated the effect of temperature by doubling $T$. The two spectra at $T=0.31 \mathrm{~K}$ and at $T=0.62 \mathrm{~K}$ are virtually identical.

For small $n$ we observe a small spike in the ${ }^{2} \Pi_{1 / 2}$ spectrum $\left(\mathrm{D}_{1}\right.$ line). This results from the extremal point of the ${ }^{2} \Pi_{1 / 2}$ potential (which has a secondary minimum and a local maximum in between). The spike is smeared out with increasing $n$.

For small $n$, the line shape of the peak around the $\mathrm{D}_{2}$ line is fully determined by the transition to the (repulsive) ${ }^{2} \Sigma_{1 / 2}$ state. Increasing the cluster size to $n=20$, the ${ }^{2} \Pi_{3 / 2}$ line has shifted and broadened only slightly while the ${ }^{2} \Sigma_{1 / 2}$ line has shifted and broadened substantially. As a result the combined $\mathrm{D}_{2}$ has a "peak-shoulder" structure (peak: ${ }^{2} \Pi_{3 / 2}$, shoulder: ${ }^{2} \Sigma_{1 / 2}$ ). Upon further increasing $n$ this trend continues. The $\mathrm{D}_{2}$ line of our calculated spectra does not have a real double peak structure for any $n$, because of the large broadening of the ${ }^{2} \Sigma_{1 / 2}$ component.

Between the spectrum for $n=300$ and the flat film surface, we see a large difference, which means $n=300$ is still very far from the limit of a flat surface. This slow convergence with cluster size was observed also experimentally for $\mathrm{Ca}$ adsorbed on ${ }^{4} \mathrm{He}$ clusters. ${ }^{57}$

For the $D_{1}\left({ }^{2} \Pi_{1 / 2}\right)$ line at $n=300$, we obtain a shift of the peak by $\sim 30 \mathrm{~cm}^{-1}$ and a linewidth (FWHM) of $\sim 35 \mathrm{~cm}^{-1}$. This is in good agreement with experiments by Brühl et al. ${ }^{9}$ where typically $n=\mathrm{O}\left(10^{3}-10^{4}\right)$. In the spec- trum of flat ${ }^{4} \mathrm{He}$ surface, the ${ }^{2} \Pi_{1 / 2}$ is shifted by about twice as much, $\sim 55 \mathrm{~cm}^{-1}$, and with a larger linewidth. For the $D_{2}\left({ }^{2} \Pi_{3 / 2}\right)$ line for up to $n=300$, the peak is very close to the free $\mathrm{Rb}$ transition, with a linewidth (FWHM) of approximately $\sim 20 \mathrm{~cm}^{-1}$. This agrees qualitatively with Brühl et al. ${ }^{9}$ who found almost no shift and a slightly smaller linewidth. In the spectrum of flat ${ }^{4} \mathrm{He}$ surface, the ${ }^{2} \Pi_{3 / 2}$ is shifted by about $35 \mathrm{~cm}^{-1}$, with about twice the linewidth. The shoulder of the $\mathrm{D}_{2}$ line in the spectrum for $n=300$ is again in qualitative agreement with Brühl et al. ${ }^{9}$ (considering the scatter in the experimental spectrum).

We conclude that the experimental spectrum by Brühl et al. ${ }^{9}$ is very similar to our calculated spectra for $n=100$ 300 , but not to our spectra for $\mathrm{Rb}$ on a flat surface. This indicates that experiments with clusters of a few thousand ${ }^{4} \mathrm{He}$ atoms $^{9}$ are still far from the limit of a flat He surface. This interpretation of the result is consistent with the small changes we see between $n=100$ and $n=300$. For quantum Monte Carlo simulation this means that one should rather use small clusters of hundreds of ${ }^{4} \mathrm{He}$ instead of using a flat surface as approximation to large clusters. However, the disagreement between our flat surface results and experiments could also lie in one or more of the approximations that we used: the Pascale potentials for the $\mathrm{Rb}^{*}-\mathrm{He}$ dimer interaction, the DIM approximation for the interaction of $\mathrm{Rb}^{*}$ with more than one He atom, and the Lax approximation of the spectrum. Concerning the sensitivity of our results to the $\mathrm{Rb}^{*}-\mathrm{He}$ dimer interaction, we are in the progress of a systematic computational study of $\mathrm{A}^{*}-\mathrm{He}$ interactions for alkalis $\mathrm{A}=\mathrm{Li}, \ldots$, Cs.

\section{CONCLUSIONS}

In this work, we continue our quantum Monte Carlo study of $\mathrm{Rb}^{*} \mathrm{He}_{n}$ that we started in paper I. There we focused on the first electronic state, associated with the excitation to the $1^{2} \Pi_{1 / 2}$ state of $\mathrm{Rb}-\mathrm{He}$ complexes, while here we focus on the $1^{2} \Pi_{3 / 2}$ excitation. As in paper I, we use the DIM approximation to model the full interaction potential of $\mathrm{Rb}^{*} \mathrm{He}_{n}$. In contrast to the $1^{2} \Pi_{1 / 2}$ state where up to $n=7 \mathrm{He}$ atoms can be strongly bound forming the $\mathrm{Rb}^{*} \mathrm{He}_{7}$ exciplex, the $1^{2} \Pi_{3 / 2}$ state allows only $n=2$ helium atoms to be strongly attached, forming a linear $\mathrm{He}-\mathrm{Rb}^{*}-\mathrm{He}$ exciplex. Increasing the cluster size beyond $n=2$, the ground state corresponds to an asymmetric distribution of ${ }^{4} \mathrm{He}$ atoms with respect to the $\mathrm{He}-\mathrm{Rb}^{*}-$ He core exciplex. This is due to the weak attraction of the $\mathrm{Rb}^{*} \mathrm{He}_{2}$ exciplex with the nonexciplexed ${ }^{4} \mathrm{He}$ atoms, leading to a preference of the ${ }^{4} \mathrm{He}$ atoms to cluster on one side of the exciplex axis, driven by the attraction of the $\mathrm{He}-\mathrm{He}$ interaction. Thus, the ground state of $\mathrm{Rb}^{*} \mathrm{He}_{n}$ is an $\mathrm{Rb}^{*} \mathrm{He}_{2}$ exciplex on the surface of a cluster of $n-2{ }^{4} \mathrm{He}$ atoms. Like $\mathrm{Rb}$ in the electronic ground state, the $\mathrm{Rb}^{*} \mathrm{He}_{2}$ exciplex is not solvated inside a ${ }^{4} \mathrm{He}$ droplet.

The dynamics of the electronic excitation of a $\mathrm{Rb}$ atom adsorbed on a large ${ }^{4} \mathrm{He}$ droplet does not lead to a ground state configuration of $\mathrm{Rb}^{*} \mathrm{He}_{n}$. In paper I, we approximated the dynamics by a "vertical Monte Carlo transition" and found that $\mathrm{Rb}$ adsorbed on a ${ }^{4} \mathrm{He}$ film is excited to a metastable $1^{2} \Pi_{1 / 2}$ state, without the formation of an exciplex, in agreement with experimental studies. ${ }^{15}$ Here we performed a 
similar study of the $1^{2} \Pi_{3 / 2}$ excitation, and observed the formation a $\mathrm{Rb}^{*} \mathrm{He}_{1}$ exciplex. Thus the potential energy surface has no large barrier to the exciplex formation and that it is reasonable to conclude that no metastable state similar to the one observed in Ref. 15 exists. $\mathrm{Rb}^{*} \mathrm{He}_{2}$ exciplexes are not obtained within this vertical transition approach. We find that both $\mathrm{Rb}^{*} \mathrm{He}_{1}$ and $\mathrm{Rb}^{*} \mathrm{He}_{2}$ would be stable against desorption from the He surface, with similar binding energies of about $10 \mathrm{~cm}^{-1}$. While our vertical MC approach cannot calculate the desorption cross section of the exciplex formation process, we note that the exciplex binding energy is much larger than this binding energy, hence desorption is energetically possible. This agrees with experimental studies of the $1^{2} \Pi_{3 / 2}$ excitation of alkali atoms on He droplets. $6,9,14,15$

The ${ }^{4} \mathrm{He}$ atom complexed with $\mathrm{Rb}^{*}$ can be considered molecule-like, since in our PIMC simulations we found no Bose exchange of this $\mathrm{He}$ atom with the other $\mathrm{He}$ atoms of the cluster or film. This leads to the general question of the role of Bose symmetry and of superfluidity in the exciplex formation process. Invoking superfluid and normal density on a microscopic scale has been very successful in the description of the effective rotational dynamics of heavy molecules in ${ }^{4} \mathrm{He} .{ }^{58-60}$ Superfluidity is a concept based on linear response theory, while exciplex formation is a highly nonlinear process. Therefore, we do not expect that exciplex formation can be understood in terms of local superfluid and normal fractions. Furthermore, we approximate the real dynamics of the process by a "vertical MC transition" which precludes the extraction of dynamical information. However, the concept of local superfluidity can be relevant for e.g., the study of the mobility of alkali atoms on the surface of ${ }^{4} \mathrm{He}$ droplets. We plan to investigate if the effective mass of heavier atoms on the surface can be related to a local normal fraction of the ${ }^{4} \mathrm{He}$ dimple formed below the adsorbed atom.

We also calculated the electronic absorption spectrum of $\mathrm{RbHe}_{n}$ in the Lax approximation, for increasing cluster size $n$ and for the limit of a flat He film surface. The convergence of the spectral shape with $n$ to the flat surface limit, $n \rightarrow \infty$, turns out to be very slow. This means that the shape of the dimple indented by the $\mathrm{Rb}$ atom in the surface of a $\mathrm{He}_{n}$ cluster converges slowly with $n$. This is not surprising: already a simple geometric construction of a dimple in a surface sphere of radius $R$ shows that the depth of the dimple is proportional to the curvature $1 / R$. Since $R \sim n^{1 / 3}$, the depth converges to the zero curvature limit of a flat surface as $1 / n^{1 / 3}$. Our results for $\mathrm{RbHe}_{300}$ indeed agree better with the experimental absorption spectra than our results for $\mathrm{Rb}$ on a $\mathrm{He}$ film.

Finally, we want to underline that we kept working with the Pascale's potentials as ingredient for the DIM matrix to be consistent with paper I. But we are aware of the criticism on the accuracy of Pascale's potentials. Efforts both in obtaining more accurate potentials and in testing the effects on $\mathrm{Rb}^{*} \mathrm{He}_{n}$ clusters are in progress.

\section{ACKNOWLEDGMENTS}

We thank J. Pascale for making the alkali-helium interaction potentials available to us and Wolfgang Ernst, Frank

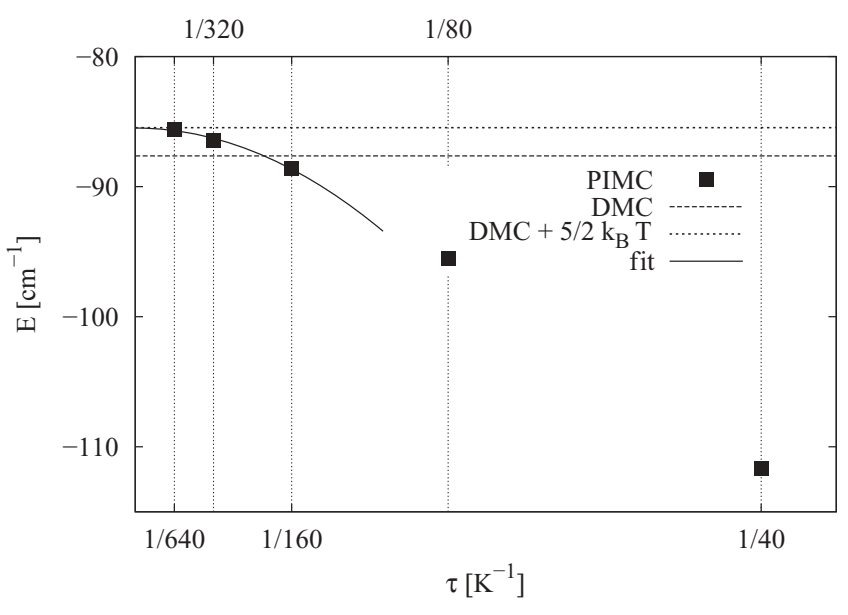

FIG. 9. Energy of $\mathrm{Rb}^{*} \mathrm{He}_{2}$ at $1.25 \mathrm{~K}$, obtained by PIMC, as function of imaginary time step $\Delta \tau$ (filled boxes; error smaller than the symbols). The full line is a quadratic fit through the lowest three data points. Also shown are the energy obtained by DMC (i.e., at $T=0 \mathrm{~K}$; dashed line) and the DMC energy plus an estimate of the thermal energy at $T=1.25 \mathrm{~K}$ (short dashed line).

Stienkemeier, and Alberto Hernando for valuable discussions. This work is supported by the Région Bretagne via the project CREATE "4023-HELIUM", by Egide via the Amadeus/Amadée PHC program No. 17283PE/FR14-2008 and by ANR via the project "DYHELIUM." M.L. acknowledges grants from CNRS and from the Academy of Finland. Grégroire Guillon and David Dell' Angelo are acknowledged for their comments on the manuscript.

\section{APPENDIX: ANALYSIS OF PIMC TIME STEP BIAS}

Since for the $\mathrm{Rb}^{*}-\mathrm{He}$ interaction we use the simple Trotter approximation of the short-time density matrix, we have to be careful with the time step bias, especially regarding the properties of the exciplex. This is because the strongly bound He atoms experience a steep potential around their equilibrium positions, thus leading to a large bias.

In Fig. 9 we show the energy of the $\mathrm{Rb}^{*} \mathrm{He}_{2}$ exciplex (where both $\mathrm{He}$ atoms are strongly bound to $\mathrm{Rb}^{*}$ ) at a temperature $T=1.25 \mathrm{~K}$, obtained by PIMC with different time steps $\Delta \tau$. For the largest time steps $\Delta \tau=(40 \mathrm{~K})^{-1}$ and $\Delta \tau$ $=(80 \mathrm{~K})^{-1}, E(\Delta \tau)$ has not even reached the quadratic behavior, therefore we made a quadratic fit using only the smallest three $\Delta \tau$. We note that the extrapolated PIMC result overshoots the DMC result for $E$ (indicated by a dashed line). This can be readily explained by the finite temperature of the PIMC simulations. We estimate the thermal effect by considering $\mathrm{Rb}^{*} \mathrm{He}_{2}$ as a classical linear rotor (with energy $k_{B} T$ ), because the rotational constant $B \approx 0.25 \mathrm{~K}$ is much smaller than $T$ and because the energies of internal vibration modes are too large to have a noticeable thermal effect at $T=1.25$ $\mathrm{K}$. Combined with the thermal translational energy $3 k_{B} T / 2$, we estimate a total thermal energy $5 k_{B} T / 2$. If we add this to the DMC result (short dashed line) we get indeed excellent agreement between DMC and PIMC.

A similar time step studied was shown in Fig. 4 of paper I in which no thermal correction to the DMC value was added. The agreement between PIMC and DMC energies for $\mathrm{Rb}^{*} \mathrm{He}_{7}$ found at that time turns out to be a fortuitous cancella- 


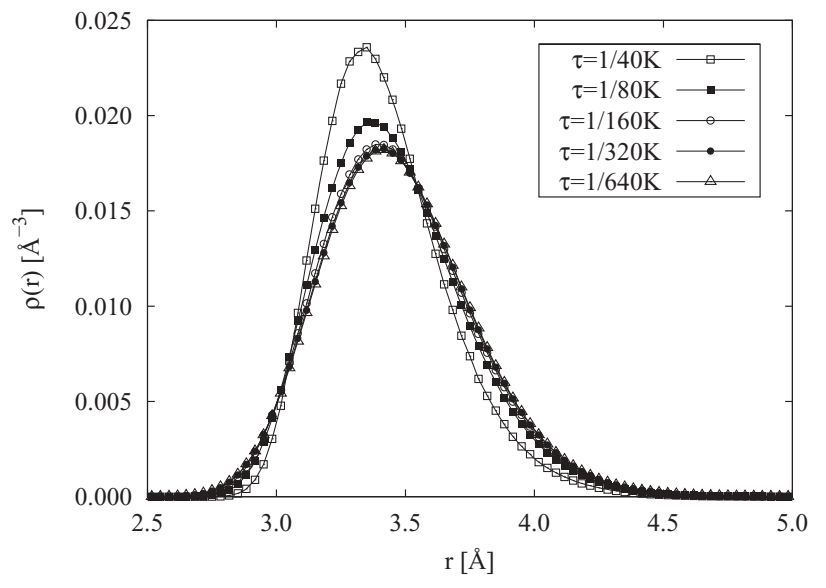

FIG. 10. He density relative to $\mathrm{Rb}^{*}$ for $\mathrm{Rb}^{*} \mathrm{He}_{2}$ at $1.25 \mathrm{~K}$, obtained by PIMC with several time steps between $\Delta \tau=(40 \mathrm{~K})^{-1}$ and $(640 \mathrm{~K})^{-1}$. Except for the two largest $\Delta \tau$ values, the results for the three smaller ones are almost indistinguishable on the scale of the figure.

tion of neglected effects. Indeed, the DMC energy of -539.5 $\pm 0.3 \mathrm{~cm}^{-1}$ was not corrected for the ensemble size bias. This bias is negligible for the smaller clusters sizes (below $1 \mathrm{~cm}^{-1}$ for clusters with $n<5$ ) but corresponds to a lowering of the energy to $-542.6 \pm 0.4 \mathrm{~cm}^{-1}$ for $\mathrm{Rb}^{*} \mathrm{He}_{7}$. The thermal effect for the quite rigid $\mathrm{He}$ ring of the $\mathrm{Rb}^{*} \mathrm{He}_{7}$ cluster can be estimated in a similar fashion as here to be $5 k_{B} T / 2$ if only two rotations are taken into account (neglecting the rotation of bosonic ${ }^{4} \mathrm{He}$ atoms around the axis of the ring they form). Thus, adding the thermal effect and correcting from the previously neglected ensemble size effect, the agreement between PIMC and DMC is recovered with an uncertainty of about $1 \mathrm{~cm}^{-1}$. Accounting for all internal degrees of freedom that could be populated at the temperature of the PIMC simulation would reduce the small remaining difference between DMC and PIMC further.

In Fig. 10 we show the ${ }^{4} \mathrm{He}$ density relative to $\mathrm{Rb}^{*}$ for $\mathrm{Rb}^{*} \mathrm{He}_{2}$ at a temperature $T=1.25 \mathrm{~K}$, again obtained with different time steps $\Delta \tau$. For $\Delta \tau=(40 \mathrm{~K})^{-1}$, the width of the density distribution is significantly underestimated. We deemed such a $\Delta \tau$ value too large for simulations which involves exciplexes if anything but qualitative results are required. For $\Delta \tau=(80 \mathrm{~K})^{-1}$ the peak density is still a bit too high, but the overall width is already close to the results for smaller values $\Delta \tau=(160 \mathrm{~K})^{-1},(320 \mathrm{~K})^{-1},(640 \mathrm{~K})^{-1}$, which are almost indistinguishable on the scale of the figure. Therefore, we consider $\Delta \tau=(80 \mathrm{~K})^{-1}$ as a compromise between accuracy and computational efficiency, the latter being an important issue for simulations of large systems (such as a He film) or at low temperature.

${ }^{1}$ J. P. Toennies and A. F. Vilesov, Angew. Chem., Int. Ed. 43, 2622 (2004).

${ }^{2}$ M. Barranco, R. Guardiola, S. Hernandez, R. Mayol, J. Navarro, and M. Pi, J. Low Temp. Phys. 142, 1 (2006).

${ }^{3}$ F. Stienkemeier and K. K. Lehmann, J. Phys. B 39, R127 (2006).

${ }^{4}$ F. Stienkemeier, J. Higgins, C. Callegari, S. I. Kanorsky, W. E. Ernst, and G. Scoles, Z. Phys. D 38, 253 (1996).

${ }^{5}$ F. Ancilotto, E. Cheng, M. W. Cole, and F. Toigo, Z. Phys. B 98, 323 (1995).

${ }^{6}$ J. Reho, C. Callegari, J. Higgins, W. E. Ernst, K. K. Lehmann, and G. Scoles, Faraday Discuss. 108, 161 (1997).
${ }^{7}$ J. Reho, J. Higgins, C. Callegari, K. K. Lehmann, and G. Scoles, J. Chem. Phys. 113, 9686 (2000).

${ }^{8}$ J. Reho, J. Higgins, K. K. Lehmann, and G. Scoles, J. Chem. Phys. 113, 9694 (2000).

${ }^{9}$ F. R. Brühl, R. A. Trasca, and W. E. Ernst, J. Chem. Phys. 115, 10220 (2001).

${ }^{10}$ C. P. Schulz, P. Claas, and F. Stienkemeier, Phys. Rev. Lett. 87, 153401 (2001).

${ }^{11}$ K. Hirano, K. Enomoto, M. Kumakura, Y. Takahashi, and T. Yabuzaki, Phys. Rev. A 68, 012722 (2003).

${ }^{12}$ G. Droppelmann, O. Büermann, C. P. Schulz, and F. Stienkemeier, Phys. Rev. Lett. 93, 023402 (2004).

${ }^{13}$ M. Mudrich, F. Stienkemeier, G. Droppelmann, P. Claas, and C. P. Schulz, Phys. Rev. Lett. 100, 023401 (2008).

${ }^{14}$ K. Enomoto, K. Hirano, M. Kumakura, Y. Takahashi, and T. Yabuzaki, Phys. Rev. A 69, 012501 (2004).

${ }^{15}$ G. Auböck, J. Nagl, C. Callegari, and W. E. Ernst, Phys. Rev. Lett. 101, 035301 (2008).

${ }^{16}$ M. Leino, A. Viel, and R. E. Zillich, J. Chem. Phys. 129, 184308 (2008).

${ }^{17}$ J. Dupont-Roc, Z. Phys. B 98, 383 (1995).

${ }^{18}$ P. Moroshkin, A. Hofer, D. Nettels, S. Ulzega, and A. Weis, J. Chem. Phys. 124, 024511 (2006).

${ }^{19}$ A. Hofer, P. Moroshkin, D. Nettels, S. Ulzega, and A. Weis, Phys. Rev. A 74, 032509 (2006).

${ }^{20}$ A. B. Pacheco, B. Thorndyke, A. Reyes, and D. A. Micha, J. Chem. Phys. 127, 244504 (2007).

${ }^{21}$ T. Takayanagi and M. Shiga, Phys. Chem. Chem. Phys. 6, 3241 (2004).

${ }^{22}$ A. Pifrader, O. Allard, G. Auböck, C. Callegari, W. E. Ernst, R. Hubert and F. Ancilotto, J. Chem. Phys. 133, 164502 (2010)..

${ }^{23}$ D. Nettels, A. Hofer, P. Moroshikin, R. Müller-Siebert, S. Ulzega, and A. Weis, Phys. Rev. Lett. 94, 063001 (2005).

${ }^{24}$ P. Moroshkin, A. Hofer, V. Lebedev, and A. Weis, Phys. Rev. A 78, 032501 (2008).

${ }^{25}$ Y. Takahashi, K. Sano, T. Kinoshita, and T. Yabuzaki, Phys. Rev. Lett. 71, 1035 (1993).

${ }^{26}$ T. Kinoshita, Y. Takahashi, and T. Yabuzaki, Phys. Rev. B 49, 3648 (1994).

${ }^{27}$ T. Kinoshita, K. Fukuda, Y. Takahashi, and T. Yabuzaki, Phys. Rev. A 52, 2707 (1995).

${ }^{28}$ Moritz Theisen, Florian Lackner, and Wolfgang E. Ernst, Phys. Chem. Chem. Phys. 12, 14861-14863 (2010).

${ }^{29}$ C. Callegari, J. Higgins, F. Stienkemeier, and G. Scoles, J. Phys. Chem. A 102, 95 (1998).

${ }^{30}$ O. Bünermann, G. Droppelmann, A. Hernando, R. Mayol, and F. Stienkemeier, J. Phys. Chem. A 111, 12684 (2007).

${ }^{31}$ M. Koch, C. Callegari, and W. E. Ernst, Mol. Phys. 109, 1005 (2010).

${ }^{32}$ L. C. Balling and J. J. Wright, J. Chem. Phys. 79, 2941 (1983).

${ }^{33}$ A. Nakayama and K. Yamashita, J. Chem. Phys. 114, 780 (2001).

${ }^{34}$ R. A. Aziz, F. R. W. McCourt, and C. C. K. Wong, Mol. Phys. 61, 1487 (1987).

${ }^{35}$ J. Pascale, Phys. Rev. A 28, 632 (1983).

${ }^{36}$ J. Pascale, Technical Report, Service de Physique des Atomes et des Surfaces (C.E.N. Saclay), Gif sur Yvette, France, 1983.

${ }^{37}$ S. A. Chin, Phys. Rev. A 42, 6991 (1990).

${ }^{38}$ J. Boronat and J. Casulleras, Phys. Rev. B 49, 8920 (1994).

${ }^{39}$ D. Blume, M. Lewerenz, F. Huisken, and M. Kaloudis, J. Chem. Phys. 105, 8666 (1996).

${ }^{40}$ A. Viel, M. D. Coutinho-Neto, and U. Manthe, J. Chem. Phys. 126, 024308 (2007).

${ }^{41}$ J. H. Hetherington, Phys. Rev. A 30, 2713 (1984).

${ }^{42}$ N. Cerf and O. C. Martin, Phys. Rev. E 51, 3679 (1995).

${ }^{43}$ M. Lewerenz, private communication (2003).

${ }^{44}$ C. J. Umrigar, M. P. Nightingale, and K. J. Runge, J. Chem. Phys. 99, 2865 (1993).

${ }^{45}$ M. P. Nightingale and H. W. J.Blöte, Phys. Rev. Lett. 60, 1562 (1988).

${ }^{46}$ C. A. Brindle, M. R. Prado, K. C. Janda, N. Halberstadt, and M. Lewerenz, J. Chem. Phys. 123, 064312 (2005).

${ }^{47}$ D. M. Ceperley, Rev. Mod. Phys. 67, 279 (1995).

${ }^{48}$ R. E. Zillich, F. Paesani, Y. Kwon, and K. B. Whaley, J. Chem. Phys. 123, 114301 (2005).

${ }^{49}$ Such an asymmetric configuration is still symmetric in the sense that the $\mathrm{He}$ atoms would aggregate on both ends of $\mathrm{He}-\mathrm{Rb}^{*}-\mathrm{He}$ with equal probability - thus fulfilling the symmetry requirements of the ground state of the symmetric Hamiltonian. 
${ }^{50}$ M. Lewerenz, J. Chem. Phys. 106, 4596 (1997).

${ }^{51}$ S. A. Chin and E. Krotscheck, Phys. Rev. B 52, 10405 (1995).

${ }^{52}$ S.Kilić, E. Krotscheck, and R. Zillich, J. Low Temp. Phys. 116, 245 (1999).

${ }^{53}$ In paper I we set the temperature to a lower value of $T=0.31 \mathrm{~K}$, because we could use a smaller time step of $\Delta \tau=(40 \mathrm{~K})^{-1}$.

${ }^{54}$ M. Lax, J. Chem. Phys. 20, 1752 (1952).
${ }^{55}$ E. Cheng and K. B. Whaley, J. Chem. Phys. 104, 3155 (1996).

${ }^{56}$ A. Hernando, M. Barranco, R. Mayol, M. Pi, and M. Krosnicki, Phys. Rev. B 77, 024513 (2008).

${ }^{57}$ F. Stienkemeier, F. Meier, and H. Lutz, J. Chem. Phys. 107, 10816 (1997).

${ }^{58}$ Y. Kwon and K. B. Whaley, Phys. Rev. Lett. 83, 4108 (1999).

${ }^{59}$ Y. Kwon and K. B. Whaley, J. Chem. Phys. 115, 10146 (2001).

${ }^{60}$ F. Paesani, Y. Kwon, and K. B. Whaley, Phys. Rev. Lett. 94, 153401 (2005). 